\title{
WAVE LENGTHS AND ZEEMAN EFFECTS IN LANTHANUM SPECTRA
}

\author{
By William F. Meggers
}

\section{ABSTRACT}

The wave lengths corresponding to more than 1,500 lines photographed in the arc and spark spectra of lanthanum were measured relative to standards in the iron spectrum. The values extend from 2,142.81 A in the ultra-violet to 10,954.6 $A$ in the infra-red. Data on the furnace spectra of 695 lanthanum lines studied by King and Carter in the interval 2,798 to 8,346 A are quoted. Measurements of Zeeman effects for 476 lines ranging in wave length from 2,791 to 7,483 A are also presented. Comparison of relative intensities and other characteristics of lines in the different sources permitted a sharp discrimination between three classes of lines; about 700 are ascribed to neutral atoms (constituting the La I spectrum), 800 originate with singly ionized atoms (La II spectrum), and 10 belong to doubly ionized atoms (La III spectrum). These data form a complete and accurate description of atomic lanthanum spectra, suitable for chemical indentification and for analysis of the spectral structures. A new infra-red sequence of La O bands is appended.

\section{CONTENTS}

II. Introduction

1. Wave lengths

2. Zeeman effects.... 240

III. Results

\section{INTRODUCTION}

Lanthanum ( $\mathrm{La}=138.90 ; \mathrm{Z}=57)$ is the third element in the sixth period of the periodic classification, and is analogous to scandium and yttrium which appear in similar positions in the fourth and fifth periods. The emission spectra of scandium ${ }^{1}$ and yttrium ${ }^{2}$ have been described with satisfactory thoroughness and precision, but lanthanum data of the same quality have been lacking up to the present.

Attempts to analyze the structures of lanthanum spectra, ${ }^{3}$ when based upon the published wave lengths and Zeeman effects, were only partially successful and it was evident that more extensive and accurate descriptive material was required before the structural analyses could be completed. Such data are now presented in this paper; they comprise wave-length measurements from 2,100 to 11,000 A, estimated relative intensities for 1,535 lines, and Zeeman effect observations for 476 lines in the interval 2,800 to $7,500 \mathrm{~A}$.

\footnotetext{
1 W. F. Meggers, B. S. Sci. Paper No. 549, vol. 22, p. 61, 1927.

W. F. Meggers, B. S. Jour. Research, vol. 1 (RP12), p. 319, 1928.

${ }^{3}$ W. F. Meggers, La I, J. Wash. Acad. Sci., vol. 17, p. 25, 1927. W. F. Meggers, La II, J. Opt. Soc. Am., vol. 14, p. 191, 1927.
} 
Analysis of the structures of lanthanum spectra and details concerning the hyperfine structure of lanthanum lines will be given in other papers.

The only remaining spectra of this type are those associated with actinium, the optical spectra of which have never been investigated.

\section{EXPERIMEN'TAL}

\section{WAVE LENGTHS}

The lanthanum salts used in this investigation consisted of $\mathrm{LaCl}_{2}$ purified by Auer $\nabla$. Welsbach, pure $\mathrm{LaO}_{3}$ kindly supplied by Prof. B. S. Hopkins of the University of Illinois, and commercial $\mathrm{LaCl}_{2}$ purchased from Eimer and Amend. The latter material contained a considerable amount of cerium, and its use was confined mainly to the observation of Zeeman effects.

Arc spectrograms were made with electric arcs between electrodes of graphite, silver, or copper, a small portion of lanthanum salt being placed on the lower electrode before striking the arc. The arc was fed by 5 to 6 amperes direct current from a circuit with 220 volts potential difference. Some of the spark spectrograms were made with the same metal electrodes which had been used previously for arc spectra, the salt fused on the rods in the arc sufficed to give welldeveloped spark spectra. Additional spark spectrograms were obtained with graphite electrodes upon which a solution of $\mathrm{LaCl}_{2}$ was allowed to drip during the exposure. For excitation of spark spectra a 40,000-volt transformer consuming about a kilowatt was used, with condensers of $0.006 \mathrm{H} \mu$ f capacity in parallel with the spark in the secondary circuit. Comparison arc and spark spectra of graphite, silver, and copper were photographed adjacent to those of the electrodes plus lanthanum salt so that lines due to the electrodes or to atmospheric gases could be recognized and avoided during measurement. Finally, the arc spectrum of iron was recorded alongside each lanthanum spectrogram to supply the scale of standard wave lengths from which the values for lanthanum lines were derived.

The wave-length interval from 2,500 A in the ultra-violet to 11,000 $A$ in the infra-red was investigated with concave gratings, while the shorter wave portion to $2,000 \AA$ was photographed with a quartz spectrograph. Since these spectrographs have already been described in connection with their use in the study of yttrium spectra ${ }^{4}$ further details concerning them can be dispensed with here.

Most of the spectrograms were made on photographic plates of thin glass coated with Eastman 33 emulsion, the plates being sensitized with pinaverdol, pinacyanol, dicyanin, and neocyanin to photograph the longer wave portions. The longest wave length photographed in lanthanum spectra with neocyanin-stained plates was $9,737 \mathrm{~A}$, but the infra-red limit was easily extended to $10,955 \mathrm{~A}$ during the past winter by employing Eastman "Q" type plates sensitized with xenocyanine.

\section{ZEEMAN EFFECTS}

Spectrograms for the study of lanthanum lines in a magnetic field were made with one of the stigmatic concave-grating spectographs 
referred to above. The spectral range investigated extended from $2,500 \mathrm{~A}$ to $8,000 \mathrm{~A}$, the first half of which was photographed in the second-order spectrum with a scale of $1.8 \mathrm{~A}$ per millimeter and the remainder in the first order. Photographic plates and procedure were the same as for wave-length measurements described above. The exposures ranged from 30 minutes in the violet to 5 hours in the red, the source being a spark between graphite electrodes upon which $\mathrm{LaCl}_{2}$ solution was allowed to drip from a burette during the exposure.

The magnetic resolution was obtained with a water-cooled Weiss electromagnet operated with a speed-regulated motor-generator set supplying 150 amperes at 98 volts to the magnet windings. With a pole gap of $6 \mathrm{~mm}$, the field strength was about 33,000 gausses. The actual field strength obtaining for each exposure was derived from the measured resolutions of standard lines. The following lines served as field standards:

\begin{tabular}{|c|c|c|}
\hline Element & Wave length & Zeeman pattern \\
\hline \multirow{2}{*}{ Ca....... } & $3,933.670$ & $\frac{\text { (1) } 3.5}{3}$ \\
\hline & $3,968.475$ & $\frac{(2) 4}{3}$ \\
\hline \multirow{2}{*}{ Al _....... } & $3,944,025$ & $\frac{(2) 4}{3}$ \\
\hline & $3,961.537$ & $\frac{\text { (1) } 3,5}{3}$ \\
\hline \multirow{3}{*}{$Z n . . .-$} & $4,680.140$ & $\frac{(0) 4}{2}$ \\
\hline & $4,722.163$ & $\frac{\text { (1) } 3,4}{2}$ \\
\hline & $4,810.534$ & $\frac{(0,1) 2,3.4}{2}$ \\
\hline \multirow{2}{*}{$\mathrm{Na}$} & $5,889.965$ & $\frac{\text { (1) } 3,5}{3}$ \\
\hline & 5, 895. 932 & $\frac{(2) 4}{3}$ \\
\hline \multirow{2}{*}{ K. } & $7,664.94$ & $\frac{\text { (1) } 3,5}{3}$ \\
\hline & $7,699.01$ & $\frac{1-14}{3}$ \\
\hline
\end{tabular}

The $\mathrm{Ca}, \mathrm{Na}$, and $\mathrm{K}$ lines arose naturally from impurities in the La $\mathrm{Cl}_{2}$ solution, and to these a 2 -minute exposure of $\mathrm{Al}$ or $\mathrm{Zn}$ was added at the end of the La exposure. Thus either four or five lines were always present to reveal the intensity of the magnetic field and the average probable error of such a determination was about one-fourth per cent.

In making the Zeeman-effect spectrograms light from the source was projected onto the slit of the spectrograph by means of a fused quartz lens after passing through a quartz Wollaston prism. A double image was thus obtained which gave a complete separation of light polarized parallel and perpendicular to the lines of force, thus permitting the two polarizations to be photographed simultaneously without overlap.

$127984-32-9$ 


\section{RESULTS}

Limited portions of the arc and spark spectra of La have already been described by different observers. The early observations up to 1910 are quoted by Kayser ${ }^{5}$; the only ones worth mentioning here are measurements in arc spectra by Rowland and Harrison $(3,104.702$ to $5,930.330 \mathrm{~A})$, and by Wolff $(2,610.428$ to $5,762.040 \mathrm{~A}){ }^{6}{ }^{6}$

A list of are lines $(2,610.43$ to $6,774.52 \mathrm{~A})$ and another of spark lines $\left(2,216.20\right.$ to $6,643.10$ A) was published by Exner and Haschek. ${ }^{7}$ The arc spectrum of La was investigated in the region of longer wave lengths $(5,501.349$ to $9,078.99 \mathrm{~A})$ by Kiess, ${ }^{8}$ and in the ultra-violet $(2,200$ to $3,100 \mathrm{~A})$ by Piña de Rubies. ${ }^{9}$

The above-mentioned descriptions refer mainly to arc spectra, they contain little or no information as to which lines belong to ionized atoms, and they cover their respective intervals with different scales of wave lengths and of intensities.

Altogether, their inadequacy as standard descriptions of the successive spectra characteristic of La atoms justifies the preparation and publication of the following results, appearing in Table 1. No mention is made in any of the earlier descriptions of La spectra of hyperfine structure; it was first noted by King ${ }^{10}$ and studied by Meggers and Burns. ${ }^{11}$ It now appears that a considerable number of the La lines are complex. (Indicated in Table 1 by the letter "c" following the wave length.) Since hyperfine structure of La lines has no particular importance in a general description, and is furthermore the subject of a special investigation, no further discussion of it will be made here; the results in Table 1, either for wave-length values or for Zeeman effects, apply to the center of gravity of a line whether it is narrow or appears widened due to partial resolution of the hyperfine

The probable errors of wave-length measurements reported in Table 1 are usually less than $0.01 \mathrm{~A}$ for the stronger lines between 3,100 and 6,000 , but the errors for the remaining lines are somewhat larger since they were measured for the most part with smaller dispersion. Many lines appearing enhanced or only on the spark spectrograms are hazy and unsymmetrical; such lines are not susceptible of precise measurement and may be in error by several hundredths of an angstrom unit.

Any attempt to describe the atomic spectra of La must contend with the nuisance of bands due to molecular compounds. In an investigation of the band spectra of $\mathrm{LaO}$, Meggers and Wheeler ${ }^{12}$ measured more than 300 band heads in the ordinary arc spectrum and classified the bands in possibly nine systems. The bands appear with considerable intensity throughout almost the entire range from 4,300 to $9,800 \mathrm{~A}$, causing difficulty and uncertainty in the detection

5 H. Kayser, Handbuch der Spectroscopie, vol. 5, p. 655, S. Hirzel, Leinzig, 1910.

6 A. Rowland and C. N. Harrison, Astrophys. J., vol. 7, p. 373, 1898; E. Wolff, Zcitschr. f. wis. Phot.,

vol. 3 , p. 395,1905

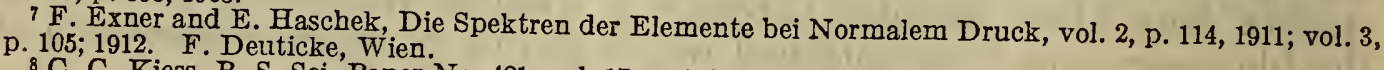

8 C. C. Kiess, B. S. Sci. Paper No. 421, vol. 17, p. 318, 1921.

${ }_{10}$ A. Piñ de Rubies, Anales Soc. Esp. Fing and E. Carter, Astrophys. J., vol. 23, p. 444, 1925.

$11 \mathrm{~W}$. S. King and E. Carter, Astrophys. J., vol. 65, p. 86, 1927.

12 W. F. Meggers and J. A. Wheeler, B. S. Jour. Research, vol. 6 (RP273), p. 239, 1931. 
of the weaker atomic lines. Since the data for LaO band heads $(3,565$ to $9,150 \mathrm{~A}$ ) have already been published, they will be omitted here, and no reference to bands will be found in Table 1 except when coincident or unresolved from lines belonging to the atomic spectra. Observations of the arc spectrum of La in the infra-red with xenocyanine plates revealed another long sequence of band heads extending from 9,111 to 9,729 A. These constitute the 0-2 sequence of System VII, and are presented here as Table 2.

The only published Zeeman effects of lanthanum lines are those observed by Rybar ${ }^{13}$ in 1911 ; they extend over the range $2,791 \mathrm{~A}$ to $5,188 \mathrm{~A}$ and include about 215 lines. These observations are fairly satisfactory, and it was at first my intention only to supplement them with data for the longer waves. However, a trial exposure in the ultra-violet indicated that Rybar's results might be improved and extended by reobservation, and the desirability of having the magnetic resolutions of La lines strictly comparable over a wide range of spectrum finally persuaded me to extend measurements to the same ultra-violet limit. In column 5 of Table 1, the observed Zeeman effects are given for 460 La lines ranging in wave length from $2,798 \mathrm{~A}$ to $7,483 \mathrm{~A}$. A few lines observed by Rybar, but not measurable on my spectrograms, are quoted and followed by the letter $R$. The patterns are presented in the standard notation for Zeeman effects; that is, the separations are expressed in decimal parts of $a$, the separation of a normal triplet, components polarized parallel to the magnetic field being inclosed in parentheses followed by the perpendicular components. In resolved complex patterns the strongest components are printed in bold-face type. For unresolved patterns a conscious effort was made to measure the strongest components when the type could be easily recognized and a symbol added to indicate the intensity distribution among the fused components. For this purpose, the notation used by Back $^{14}$ for distinguishing various types of intensity gradients was employed. The letters A or B after a Zeeman effect mean that the pattern is complex but unresolved, and A indicates that the maximum intensity for perpendicular components is at the edge of a group, while B signifies that it is in the middle. The distinction between strongest component inside or outside of the group is shown by $\mathrm{A}^{1}$ and $\mathrm{A}^{2}$, respectively. The interpretation of these Zeeman effects will be given in another paper dealing with the spectral series classification of La lines.

An important contribution to the description of the atomic spectra of La is found in the temperature classification of $\mathrm{La}$ lines by King and Carter. ${ }^{15}$ From an examination of the furnace, arc, and spark spectra in the interval 2,800 A to $8,400 \mathrm{~A}$ data were obtained for a classification of $695 \mathrm{La}$ lines according to the temperature required for initial appearance and the rate of change of line intensity as the temperature is increased. These lines were divided into five classes. Lines in Classes I and II appear at low temperature $\left(2,000^{\circ} \mathrm{C}\right.$.), but those of Class I show a slower change from low to high temperature

13 S. Rybar, Math. es Phys. Lapok, vols. 20-21, p. 198, 1911-12; Phys. Zeitschr. vol. 12, p. 889, 1911, a partial list.

14 E. Back, Zeitschr. f. Phys., vol. 15, p. 212, 1923.

15 A. S. King and E. Carter, Astrophys. J. 65, p. 86; 1927. 
than those of Class II, and as a rule are less conspicuous in the arc. Lines of Class III are usually well developed at medium temperature $\left(2,200^{\circ}\right.$ to $2,300^{\circ}$ C. $)$, while lines clearly associated with high temperatures $\left(2,600^{\circ}\right.$ to $2,800^{\circ}$ C. $)$ are placed in Classes IV and V, those of Class $V$ being absent or very faint in the furnace spectrum. A large number (230) of the lines thus classified are enhanced in the spark and more than half of these appear in the furnace. This indicates that La atoms are ionized with relative ease, which accounts for the great prominence of spark lines in the ordinary arc. In fact, a majority of the La atoms appear to be ionized in the 220-volt arc, and there can be no doubt that the best method of developing the spectrum of neutral atoms is by means of a temperature-controlled vacuum furnace which restricts ionization and also eliminates confusion with molecular spectra. A few lines not detected on my spectrograms were measured on furnace spectrograms by Professor Russell; they are marked H. N. R. in Table 1.

In the last column of Table 1 an attempt is made to assign each observed wave length to its proper atomic origin. This separation of lines into La I, La II, La III spectra is based primarily upon the relative intensities and other characteristics appearing in column 2. It is supported by the temperature classification and further description in column 3 and by Zeeman effects (column 5) describing combinations of spectral terms having even multiplicity for La I and La III lines, but odd multiplicity for La II lines. In the infra-red where data on spark intensities, temperature classes, and Zeeman effects are lacking, the lines are assumed to belong to the La I spectrum unless they are accounted for as combinations of the La II spectral terms. The significance of the symbols and abbreviations used in Table 1 is summarized as follows:

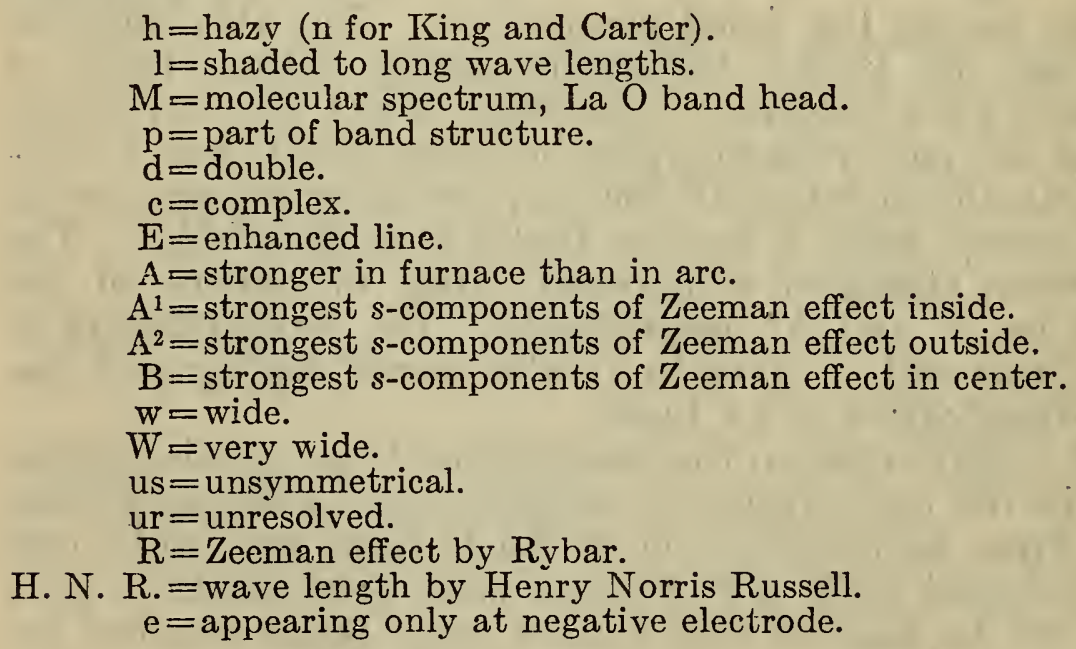


TABLE 1.-Wave lengths and Zeeman effects in lanthanum spectra

\begin{tabular}{|c|c|c|c|c|c|}
\hline $\begin{array}{c}\lambda \text { (air) } \\
\text { I. A. }\end{array}$ & $\begin{array}{c}\text { Intensity } \\
\text { arc spark } \\
\text { B. S. }\end{array}$ & $\begin{array}{c}\text { Arc intensity } \\
\text { and tempera- } \\
\text { ture class } \\
\mathrm{K} \text { and } \mathrm{C}\end{array}$ & $\begin{array}{c}\nu(\text { vac. }) \\
\mathrm{cm}^{-1}\end{array}$ & Zeeman effect & $\begin{array}{l}\text { Spec- } \\
\text { trum }\end{array}$ \\
\hline $\begin{array}{l}10,954.6 \\
10,952.0 \\
10,739.66 \\
10,612.56 \\
10,552.41\end{array}$ & $\begin{array}{r}3 \\
1 \\
5 \\
10 \\
6\end{array}$ & & $\begin{array}{l}9,126.09 \\
9,128.25 \\
9,303.73 \\
9,420.21 \\
9,473.91\end{array}$ & & $\begin{array}{l}\text { II } \\
\text { I } \\
\text { I } \\
\text { I }\end{array}$ \\
\hline $\begin{array}{c}10,522.09 \\
10,483.0 \\
61.69 \\
50.82 \\
23.4\end{array}$ & $\begin{array}{r}10 \\
2 \\
15 \\
20 \\
1\end{array}$ & & $\begin{array}{r}9,501.21 \\
36.6 \\
56.07 \\
66.01 \\
9,591.2\end{array}$ & & $\begin{array}{l}\text { I } \\
\text { I } \\
\text { I } \\
\text { I } \\
\text { I }\end{array}$ \\
\hline $\begin{array}{r}10,409.55 \\
10,372.4 \\
57.70 \\
49.08 \\
37.20\end{array}$ & $\begin{array}{r}3 \\
1 \\
20 \\
40 \\
3\end{array}$ & & $\begin{array}{r}9,603.93 \\
33.4 \\
52.01 \\
60.05 \\
71.15\end{array}$ & & $\begin{array}{l}\text { I } \\
\text { I } \\
\text { I } \\
\text { I } \\
\text { I }\end{array}$ \\
\hline $\begin{array}{r}32.40 \\
30.3 \\
10,318.2 \\
10,294.68 \\
85.64\end{array}$ & $\begin{array}{r}2 \\
1 \\
2 \\
10 \mathrm{~d} \\
3\end{array}$ & & $\begin{array}{r}75.64 \\
77.6 \\
9,689.0 \\
9,711.09 \\
21.52\end{array}$ & & $\begin{array}{l}\text { I } \\
\text { I } \\
\text { I } \\
\text { I } \\
\text { I }\end{array}$ \\
\hline $\begin{array}{l}81.34 \\
78.52 \\
74.85 \\
34.78 \\
23.76\end{array}$ & $\begin{array}{r}10 \\
3 \\
10 \\
2 \\
1\end{array}$ & & $\begin{array}{l}23.69 \\
26.37 \\
29.84 \\
67.93 \\
78.46\end{array}$ & & $\begin{array}{l}\text { I } \\
\text { I } \\
\text { I } \\
\text { I } \\
\text { I }\end{array}$ \\
\hline $\begin{array}{r}19.83 \\
10.209 .85 \\
10,186.5 \\
84.60 \\
77.74\end{array}$ & $\begin{array}{r}3 \\
2 \\
2 \\
20 \\
6 \mathrm{~h}\end{array}$ & & $\begin{array}{r}82.22 \\
9,791.78 \\
9,814.2 \\
16.06 \\
22.67\end{array}$ & & $\begin{array}{l}\text { I } \\
\text { I } \\
\text { II } \\
\text { I }\end{array}$ \\
\hline $\begin{array}{r}54.74 \\
43.38 \\
41.20 \\
30.82 \\
10,111.9\end{array}$ & $\begin{array}{r}40 \\
2 \\
10 \\
5 \\
2 \mathrm{~h}\end{array}$ & & $\begin{array}{r}44.92 \\
55.95 \\
58.06 \\
68.17 \\
9,886.6\end{array}$ & & $\begin{array}{l}\text { I } \\
\text { I } \\
\text { I } \\
\text { I } \\
\text { I }\end{array}$ \\
\hline $\begin{array}{r}10,093.54 \\
83.96 \\
66.77 \\
53.79 \\
54.82\end{array}$ & $\begin{array}{l}1 \\
2 \\
6 \\
2 \\
2\end{array}$ & & $\begin{array}{r}9,904.62 \\
14.03 \\
30.95 \\
34.84 \\
42.76\end{array}$ & & $\begin{array}{l}\text { II } \\
\text { II } \\
\text { I } \\
\text { I }\end{array}$ \\
\hline $\begin{array}{r}29.74 \\
14.45 \\
10,005.73 \\
9,988.47 \\
81.24\end{array}$ & $\begin{array}{r}2 \\
4 \\
50 \\
10 \\
6\end{array}$ & & $\begin{array}{r}67.62 \\
82.84 \\
9,991.54 \\
10,008.80 \\
016.05\end{array}$ & & $\begin{array}{l}\text { I } \\
\text { I } \\
\text { I } \\
\text { I } \\
\text { I }\end{array}$ \\
\hline $\begin{array}{r}80.38 \\
65.70 \\
32.72 \\
20.82 \\
9,911.08\end{array}$ & $\begin{array}{r}10 \\
3 \\
2 \\
150 \\
3\end{array}$ & & $\begin{array}{l}016.91 \\
031.67 \\
064.98 \\
077.06 \\
086.95\end{array}$ & & $\begin{array}{l}\text { I } \\
\text { I } \\
\text { I } \\
\text { I } \\
\text { I }\end{array}$ \\
\hline $\begin{array}{r}9,893.82 \\
81.24 \\
62.60 \\
52.58 \\
48.70\end{array}$ & $\begin{array}{r}4 \\
100 \\
3 \\
6 \\
4\end{array}$ & & $\begin{array}{l}104.55 \\
117.42 \\
136.54 \\
146.85 \\
150.84\end{array}$ & & $\begin{array}{l}\text { II } \\
\text { I } \\
\text { I } \\
\text { I }\end{array}$ \\
\hline $\begin{array}{r}42.0 \\
3.30 \\
05.2 \\
9,804.20 \\
9,775.09\end{array}$ & $\begin{array}{r}2 \\
3 h \\
1 h \\
2 \\
8\end{array}$ & & $\begin{array}{l}157.8 \\
166.74 \\
195.9 \\
196.92 \\
227.28\end{array}$ & - & $\begin{array}{l}\text { I } \\
\text { I } \\
\text { I } \\
\text { I } \\
\text { I }\end{array}$ \\
\hline $\begin{array}{r}72.24 \\
68.82 \\
37.09 \\
13.52 \\
9,709.45\end{array}$ & $\begin{array}{r}20 \\
3 h \\
100 \\
3 \\
10\end{array}$ & & $\begin{array}{r}230.26 \\
233.85 \\
267.20 \\
292.11 \\
10,296.42\end{array}$ & & $\begin{array}{l}\text { I } \\
\text { I } \\
\text { I } \\
\text { I }\end{array}$ \\
\hline
\end{tabular}


TABLE 1.-Wave lengths and Zeeman effects in lanthanum spectra-Continued

\begin{tabular}{|c|c|c|c|c|c|}
\hline $\begin{array}{c}\lambda \text { (air) } \\
\text { I. A. }\end{array}$ & $\begin{array}{c}\text { Intensity } \\
\text { arc spark } \\
\text { B. S. }\end{array}$ & $\begin{array}{l}\text { Arc intensity } \\
\text { and tempera- } \\
\text { ture class } \\
\mathbf{K} \text { and } \mathbf{C}\end{array}$ & $\begin{array}{c}\nu \text { (vac.) } \\
\text { cm }^{-1}\end{array}$ & Zeeman effect & $\begin{array}{l}\text { Spec- } \\
\text { trum }\end{array}$ \\
\hline $\begin{array}{c}9,706.48 \\
9,699.64 \\
96.7 \\
92.6 \\
72.94\end{array}$ & $\begin{array}{r}20 \\
20 \\
1 \\
2 \\
3\end{array}$ & & $\begin{array}{c}10,299.57 \\
306.84 \\
310.0 \\
314.3 \\
335.29\end{array}$ & & $\begin{array}{l}\text { I } \\
\text { I } \\
\text { I } \\
\text { I } \\
{ }_{\text {II }}\end{array}$ \\
\hline $\begin{array}{l}72.04 \\
57.00 \\
46.47 \\
40.81 \\
33.72\end{array}$ & $\begin{array}{r}8 \\
20 \\
3 \\
20 \\
40\end{array}$ & & $\begin{array}{l}336.25 \\
352.35 \\
363.65 \\
369.73 \\
377.36\end{array}$ & . & $\begin{array}{l}\text { I } \\
I_{\text {II }} \\
\text { I }\end{array}$ \\
\hline $\begin{array}{r}9,631.84 \\
9,510.38 \\
63.60 \\
60.69 \\
42.06\end{array}$ & $\begin{array}{r}2 \\
5 \\
4 \\
10 \\
50\end{array}$ & & $\begin{array}{l}379.38 \\
446.04 \\
453.45 \\
456.63 \\
477.05\end{array}$ & & $\begin{array}{l}\text { I } \\
\text { I } \\
\text { II } \\
\text { I }\end{array}$ \\
\hline $\begin{array}{r}41.23 \\
9,528.0 \\
9,485.15 \\
84.2 \\
76.98\end{array}$ & $\begin{array}{r}20 \\
1 h \\
15 \\
1 \\
3\end{array}$ & & $\begin{array}{l}477.96 \\
492.5 \\
539.91 \\
541.0 \\
548.99\end{array}$ & & $\begin{array}{l}\text { I } \\
\text { I } \\
\text { I } \\
\text { I } \\
\text { I }\end{array}$ \\
\hline $\begin{array}{l}74.45 \\
67.25 \\
61.82 \\
57.62 \\
41.7\end{array}$ & $\begin{array}{r}5 \\
2 \\
60 \\
2 \\
1\end{array}$ & & $\begin{array}{l}551.81 \\
559.84 \\
565.89 \\
570.59 \\
588.4\end{array}$ & & $\begin{array}{l}\text { I } \\
\text { I } \\
\text { I } \\
\text { I } \\
\text { I }\end{array}$ \\
\hline $\begin{array}{r}38.30 \\
15.64 \\
9,412.65 \\
9,398.2 \\
90.56\end{array}$ & $\begin{array}{r}100 \\
3 \\
100 \\
1 \\
4\end{array}$ & . & $\begin{array}{l}592.23 \\
617.71 \\
621.08 \\
637.4 \\
646.07\end{array}$ & & $\begin{array}{l}\text { I } \\
\text { I } \\
\text { I } \\
\text { I } \\
\text { I }\end{array}$ \\
\hline $\begin{array}{l}77.71 \\
76.10 \\
72.57 \\
46.69 \\
28.87\end{array}$ & $\begin{array}{r}3 \\
3 \\
30 \\
15 \\
2\end{array}$ & & $\begin{array}{l}660.66 \\
662.49 \\
661.51 \\
696.04 \\
716.47\end{array}$ & & $\begin{array}{l}\text { I } \\
\text { I } \\
\text { I } \\
\text { II }\end{array}$ \\
\hline $\begin{array}{c}24.5 \\
9,312.9 \\
9,293.3 \\
87.5 \\
60.42\end{array}$ & $\begin{array}{r}1 \\
1 \mathrm{~h} \\
2 \mathrm{~h} \\
1 \mathrm{~h} \\
3\end{array}$ & & $\begin{array}{l}721.5 \\
724.5 \\
757.5 \\
764.2 \\
795.69\end{array}$ & & $\begin{array}{l}\text { I } \\
\text { I } \\
\text { I } \\
{ }_{\text {II }}\end{array}$ \\
\hline $\begin{array}{r}54.70 \\
50.06 \\
26.63 \\
9,219.64 \\
9,172.88\end{array}$ & $\begin{array}{r}10 \\
20 \\
30 \\
10 \\
5\end{array}$ & & $\begin{array}{l}802.36 \\
807.78 \\
835.22 \\
843.44 \\
898.71\end{array}$ & & $\begin{array}{l}\text { I } \\
\text { I } \\
\text { I } \\
\text { I } \\
\text { I }\end{array}$ \\
\hline $\begin{array}{l}72.39 \\
57.13 \\
51.62 \\
46.75 \\
43.78\end{array}$ & $\begin{array}{r}10 \\
10 \\
6 \\
2 \\
5\end{array}$ & & $\begin{array}{l}899.30 \\
917.46 \\
924.03 \\
929.85 \\
933.40\end{array}$ & & $\begin{array}{l}\mathrm{I} \\
\mathrm{I} \\
\mathrm{I} \\
\mathrm{I}\end{array}$ \\
\hline $\begin{array}{r}42.24 \\
27.5 \\
19.18 \\
09.25 \\
9,101.10\end{array}$ & $\begin{array}{r}6 \\
1 \\
20 \\
2 \\
2\end{array}$ & & $\begin{array}{l}935.24 \\
952.9 \\
962.89 \\
974.84 \\
984.67\end{array}$ & & $\begin{array}{l}\mathrm{I}^{\mathrm{I}} \\
\mathrm{I}_{\mathrm{II}}\end{array}$ \\
\hline $\begin{array}{r}9,096.71 \\
79.10 \\
58.63 \\
56.53 \\
46.97\end{array}$ & $\begin{array}{r}3 \\
50 \\
2 \\
5 \\
2\end{array}$ & & $\begin{array}{r}10,989.97 \\
11,011.29 \\
036.17 \\
038.73 \\
050.39\end{array}$ & & $\begin{array}{l}\text { II }^{\text {II }} \\
\text { I } \\
\text { I }\end{array}$ \\
\hline $\begin{array}{r}25.05 \\
16.80 \\
9,008.26 \\
8,977.39 \\
8,970.07\end{array}$ & $\begin{array}{l}4 \\
2 \\
6 \\
2 \\
3\end{array}$ & & $\begin{array}{r}077.23 \\
087.37 \\
097.88 \\
136.04 \\
11,145.13\end{array}$ & & $\begin{array}{l}\text { I } \\
\text { II } \\
\text { I } \\
\text { I }\end{array}$ \\
\hline
\end{tabular}


TABLE 1.-Wave lengths and Zeeman effects in lanthanum spectra-Continued

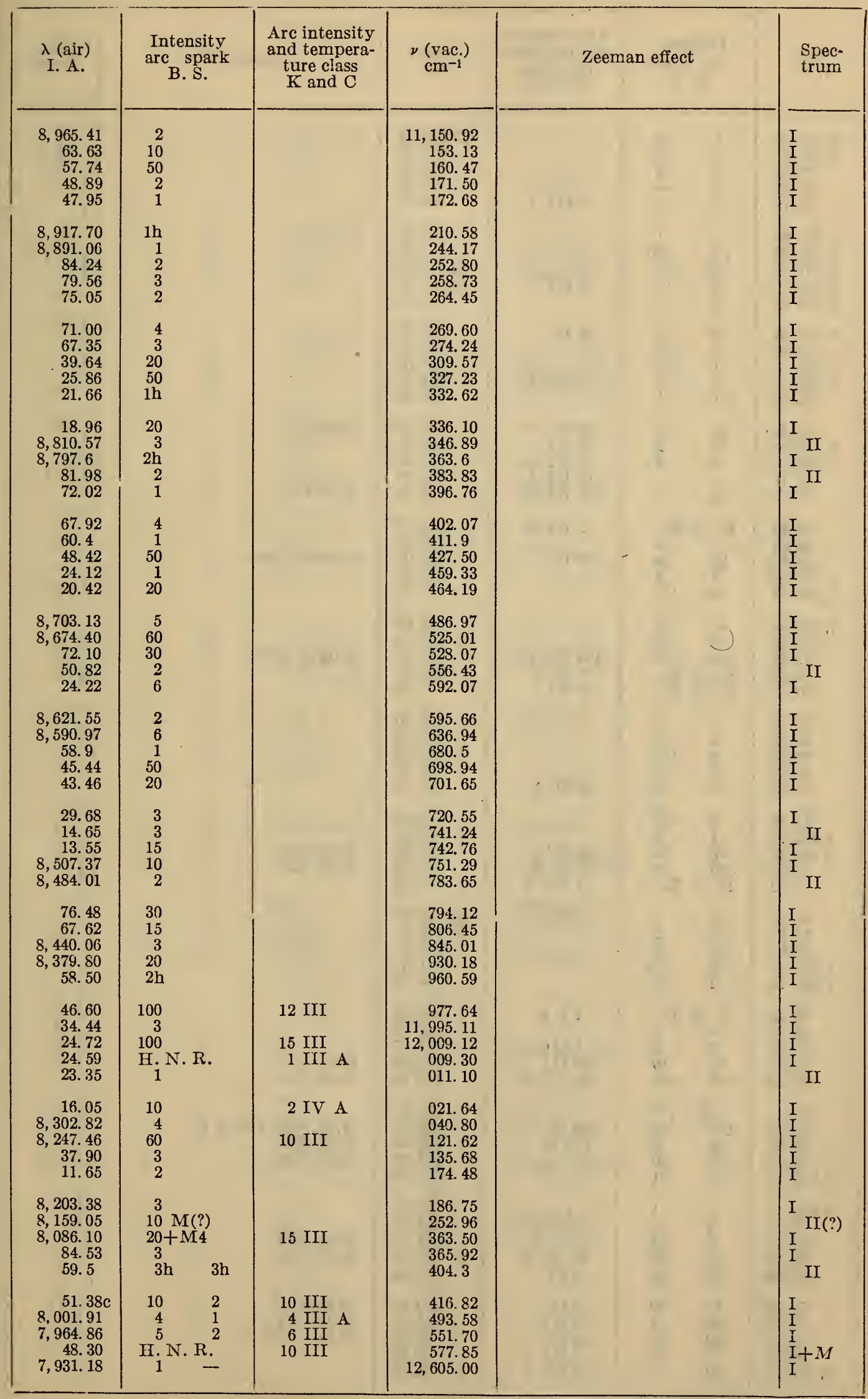


TABLE 1.-Wave lengths and Zeeman effects in lanthanum spectra-Continued

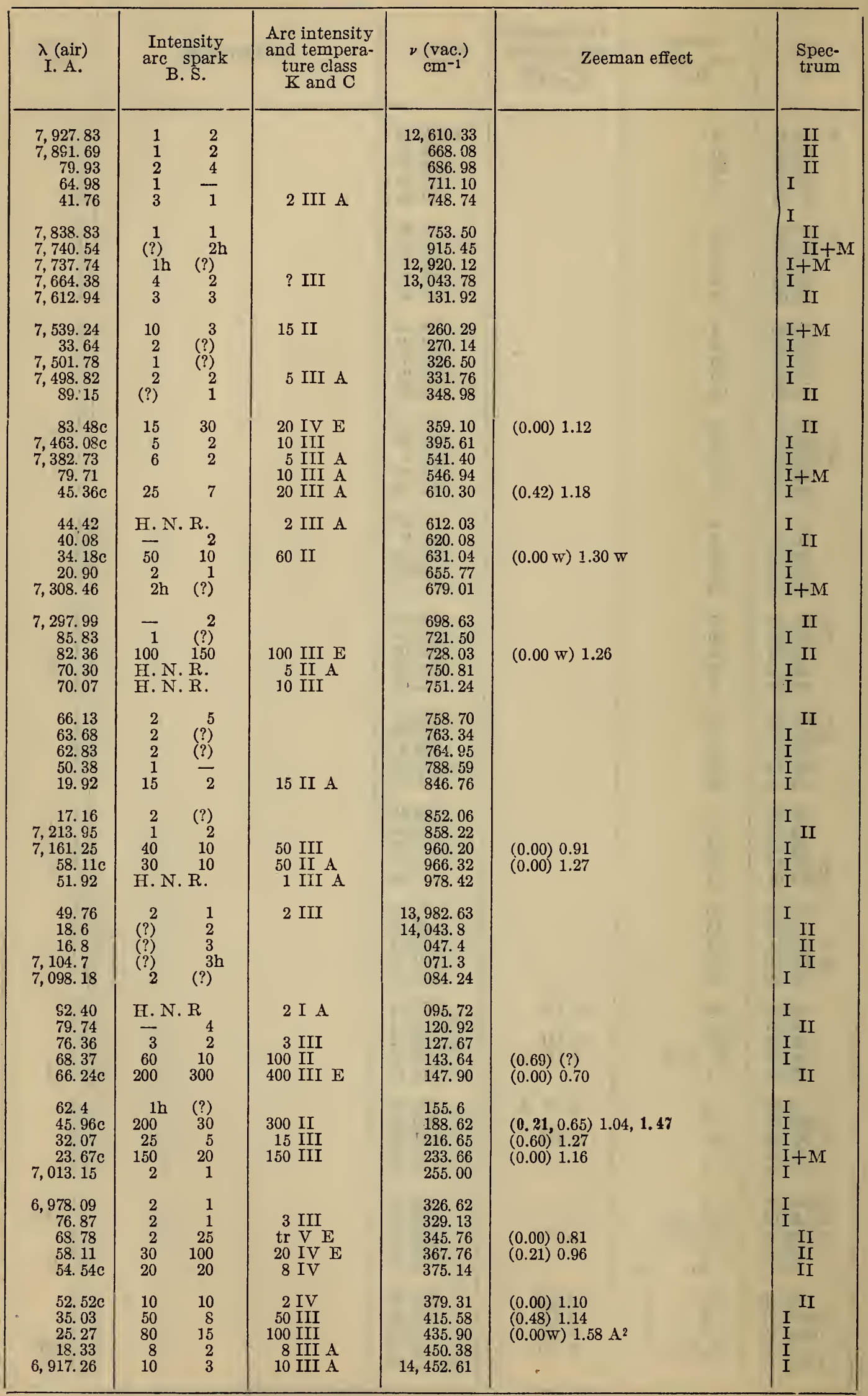


TABLE 1.-Wave lengths and Zeeman effects in lanthanum spectra-Continued

\begin{tabular}{|c|c|c|c|c|c|c|c|}
\hline $\begin{array}{c}\lambda \text { (air) } \\
\text { I. A. }\end{array}$ & $\begin{array}{l}\text { Intensity } \\
\text { arc spark } \\
\text { B. S. }\end{array}$ & $\begin{array}{l}\text { Arc intensity } \\
\text { and tempera- } \\
\text { ture class } \\
\mathrm{K} \text { and } \mathrm{C}\end{array}$ & $\begin{array}{c}\nu(\text { vac. }) \\
\mathrm{cm}^{-1}\end{array}$ & Zeeman & effect & & $\begin{array}{l}\text { Spec- } \\
\text { trum }\end{array}$ \\
\hline $\begin{array}{r}6,903.08 \\
6,902.08 \\
6,899.63 \\
98.41 \\
59.03\end{array}$ & $\begin{array}{ll}2 & 1 \\
3 & 3 \\
2 \mathrm{n} & 1 \\
& \\
4 & 5\end{array}$ & $\begin{array}{l}1 \text { III } A \\
1 \text { III } \\
\operatorname{tr} V \\
\operatorname{tr} V \\
\operatorname{tr} V\end{array}$ & $\begin{array}{r}14,482.30 \\
484.40 \\
489.54 \\
492.10 \\
575.31\end{array}$ & 5. & , & 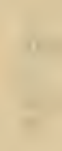 & $\begin{array}{l}\text { I } \\
\text { I(?) II } \\
I_{\text {II }}\end{array}$ \\
\hline $\begin{array}{l}37.91 \\
34.07 \\
30.83 \\
23.80 \\
21.51\end{array}$ & $\begin{array}{rr}15 & 15 \\
20 & 20 \\
1 & 6 \\
40 & 10 \\
5 \mathrm{~d} & 2\end{array}$ & $\begin{array}{l}3 \mathrm{IV}(?) \mathrm{F} \\
4 \mathrm{IV}(?) \mathrm{E} \\
\operatorname{tr} \mathrm{VE} \\
15 \mathrm{II} \mathrm{A} \\
3 \mathrm{n} \mathrm{V}\end{array}$ & $\begin{array}{l}620.32 \\
628.54 \\
635.48 \\
650.56 \\
655.47\end{array}$ & $\begin{array}{ll}(1.05) & (?) \\
(0.00) & 0.98 \\
(0.00) & 1.15\end{array}$ & 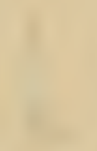 & 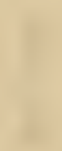 & $\mathrm{I}_{\text {I II }}^{\substack{\text { II } \\
\text { II }}}$ \\
\hline $\begin{array}{c}19.14 \\
16.29 \\
13.63 \\
03.88 \mathrm{c} \\
6,801.38\end{array}$ & $\begin{array}{rr}1 & - \\
5 & 50 \\
30 & 30 \\
1 & 5\end{array}$ & $\begin{array}{l}\ln V(?) \\
1 \mathrm{~V}(?) \mathrm{E} \\
6 \mathrm{IV}\end{array}$ & $\begin{array}{l}660.57 \\
666.70 \\
672.32 \\
682.66 \\
698.85\end{array}$ & $\begin{array}{l}(1.01) 1.29 \mathrm{~B} \\
(0.00 \mathrm{~h}) 1.56 \mathrm{~h} \\
(0.00) 1.10\end{array}$ & . & 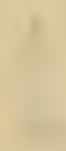 & $\begin{array}{l}\text { I } \\
\text { II } \\
\text { II } \\
\text { II }\end{array}$ \\
\hline $\begin{array}{r}6,796.73 \\
83.55 \\
78.19 \\
76.69 \\
74.28 \mathrm{c}\end{array}$ & $\begin{array}{rr}4 & 1 \\
1 & - \\
1 & - \\
1 & - \\
80 & 100\end{array}$ & 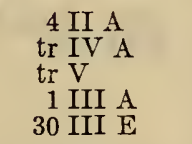 & $\begin{array}{l}708.90 \\
737.48 \\
749.14 \\
752.40 \\
757.65\end{array}$ & $(6.00 \mathrm{w}) 1.10$ & 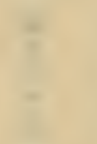 & 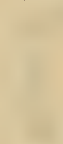 & $\begin{array}{lll}\text { I } & \\
\text { I } \\
\text { I } \\
\text { I } \\
\\
& \text { II }\end{array}$ \\
\hline $\begin{array}{l}60.73 \\
60.23 \\
53.05 \\
50.47 \\
48.12 \mathrm{c}\end{array}$ & $\begin{array}{cr}\text { H. N. R. } \\
1 & 1 \\
40 & 10 \\
1 & 1 \\
10 & 3\end{array}$ & $\begin{array}{l}1 \mathrm{III} \mathrm{A} \\
50 \mathrm{I} \mathrm{A} \\
10 \mathrm{II} \mathrm{A}\end{array}$ & $\begin{array}{l}787.23 \\
788.32 \\
804.04 \\
809.70 \\
814.86\end{array}$ & $(0.00 \mathrm{~W}) 1.51 \mathrm{~A}^{2}$ & - & $4=$ & $\begin{array}{l}\text { I } \\
\text { I } \\
\text { I. } \\
\text { II }\end{array}$ \\
\hline $\begin{array}{l}41.20 \\
37.29 \\
32.80 \mathrm{c} \\
18.68 \\
15.96\end{array}$ & $\begin{array}{l}\frac{1}{40} \\
60 \\
-\end{array}$ & $\begin{array}{l}1 \mathrm{VE} \\
\operatorname{tr} \mathrm{VE} \\
1 \mathrm{IV} \Lambda(?)\end{array}$ & $\begin{array}{l}830.07 \\
838.67 \\
848.57 \\
879.78 \\
885.80\end{array}$ & $\begin{array}{l}(0.00) \\
(0.99) \\
(0.12 \mathrm{~B}\end{array}$ & 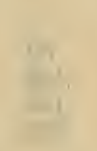 & $\mathcal{J}$ & $\begin{array}{l}\text { I } \\
\text { II } \\
\text { II }\end{array}$ \\
\hline $\begin{array}{r}14.08 \\
6,709.49 \\
6,699.86 \\
99.26 \\
92.86 \mathrm{c}\end{array}$ & $\begin{array}{r}5 \\
150 \\
3 \\
2 \\
20\end{array}$ & $\begin{array}{l}2 \mathrm{~V} \mathrm{E} \\
200 \mathrm{I} \\
4 \mathrm{III} A \\
2 \text { III(?) } \\
30 \text { I A }\end{array}$ & $\begin{array}{l}889.97 \\
900.16 \\
921.57 \\
922.91 \\
937.18\end{array}$ & $\begin{array}{l}(0.00) 1.38 \\
(0.00) 1.18 \\
(0.00) 1.06\end{array}$ & $\because$ & , & $\begin{array}{l}\text { II } \\
\text { I } \\
\text { I } \\
\text { I } \\
\text { I }\end{array}$ \\
\hline $\begin{array}{l}76.14 \\
71.41 \mathrm{c} \\
64.45 \\
61.40 \mathrm{c} \\
58.06\end{array}$ & $\begin{array}{r}1 \\
15 \\
1 \\
60 \\
1\end{array}$ & $\begin{array}{l}6 \mathrm{IV}(?) \mathrm{E} \\
80 \mathrm{I} \mathrm{A}\end{array}$ & $\begin{array}{r}974.59 \\
14,985.20 \\
15,000.85 \\
007.72 \\
015.25\end{array}$ & $\begin{array}{l}(0.63) 1.17 \\
(0.65) 1.23 \mathrm{~B}(?)\end{array}$ & 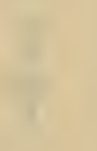 & & $\begin{array}{l}\text { II } \\
\text { II } \\
\text { I } \\
\text { I }\end{array}$ \\
\hline $\begin{array}{l}50.81 \mathrm{c} \\
45.15 \\
44.40 \mathrm{c} \\
42.79 \\
36.53\end{array}$ & $\begin{array}{rr}80 & 20 \\
7 & 3 \\
30 & 5 \\
10 & 100 \\
3 & 5\end{array}$ & $\begin{array}{c}100 \mathrm{IA} \\
8 \mathrm{IV} \\
40 \mathrm{IA} \\
3 \mathrm{VE} \\
1 \mathrm{~V}\end{array}$ & $\begin{array}{l}031.62 \\
044.42 \\
046.12 \\
049.77 \\
063.96\end{array}$ & $\begin{array}{l}(0.00 \mathrm{w}) 0.88 \\
(0.33) 0.82\end{array}$ & 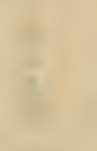 & c & $\begin{array}{l}\text { I } \\
\text { I } \\
\text { I } \\
\text { II } \\
\text { II }\end{array}$ \\
\hline $\begin{array}{l}31.20 \\
28.4 \\
19.10 \\
16.59 \mathrm{e} \\
12.48\end{array}$ & $\begin{array}{c}3 \\
\frac{1}{60} \\
3\end{array}$ & $\begin{array}{l}3 \mathrm{~V}(?) \\
1 \mathrm{IVA} \\
80 \mathrm{I}\end{array}$ & $\begin{array}{l}076.07 \\
082.4 \\
103.63 \\
109.36 \\
118.75\end{array}$ & $(0.59) 1.12 \mathrm{~B}$ & & T & $\begin{array}{l}\text { I(?) } \\
\text { I } \\
\text { II } \\
\text { I }\end{array}$ \\
\hline $\begin{array}{c}08.25 \mathrm{c} \\
07.7 \\
6,600.17 \mathrm{c} \\
6,599.48 \\
93.45 \mathrm{c}\end{array}$ & $\begin{array}{r}40 \\
1 \\
25 \\
40\end{array}$ & $\begin{array}{l}40 \text { II } \\
3 \text { IV } \\
50 \text { II A } \\
\text { tr IV A } \\
60 \text { I }\end{array}$ & $\begin{array}{l}128.43 \\
129.7 \\
146.95 \\
148.53 \\
162.39\end{array}$ & $\begin{array}{l}(0.00) 1.01 \\
(0.35) 1.12 \\
(0.00 \mathrm{w}) 1.30 \mathrm{~A}^{2}\end{array}$ & . & & $\begin{array}{l}\mathrm{I} \\
\mathrm{I}+\mathrm{M} \\
\mathrm{I}\end{array}$ \\
\hline $\begin{array}{l}90.59 \\
82.18 \\
78.51 \mathrm{c} \\
70.96 \\
63.54\end{array}$ & $\begin{array}{r}\overline{2} \\
100 \\
(?) \\
(?)\end{array}$ & $\begin{array}{c}6 \mathrm{IV} \\
400 \mathrm{I} \\
2 \mathrm{n} \mathrm{III}\end{array}$ & $\begin{array}{l}168.97 \\
188.35 \\
196.82 \\
214.28 \\
219.89\end{array}$ & $\begin{array}{l}(0.00 \mathrm{~h}) 1.41 \\
(0.00) 1.00\end{array}$ & & . & $\begin{array}{l}\text { I } \\
\text { I } \\
\text { II }\end{array}$ \\
\hline $\begin{array}{r}65.45 \\
55.95 \\
55.11 \\
54.18 \\
6,551.78\end{array}$ & $\begin{array}{ll}40 & (?) \\
& (?) \\
2 & (?) \\
1 & (?)\end{array}$ & $\begin{array}{l}40 \mathrm{I} \mathrm{A} \\
3 \mathrm{III} A \\
1 \mathrm{~V} \\
1 \mathrm{~V}\end{array}$ & $\begin{array}{r}227.05 \\
249.12 \\
251.07 \\
253.23 \\
15,258.82\end{array}$ & ins, & $\ldots$ & $\theta$ & $\begin{array}{l}\text { I } \\
1 \\
\text { I }\end{array}$ \\
\hline
\end{tabular}


TABLE 1.-Wave lengths and Zeeman effects in lanthanum spectra-Continued

\begin{tabular}{|c|c|c|c|c|c|c|}
\hline \multirow{2}{*}{$\begin{array}{c}\lambda \text { (air) } \\
\text { I. A. } \\
6,549.16 \\
43.17 \mathrm{c} \\
29.72 \mathrm{c} \\
26.99 \mathrm{c} \\
23.86\end{array}$} & \multicolumn{2}{|c|}{$\begin{array}{l}\text { Intensity } \\
\text { arc spark } \\
\text { B. S. }\end{array}$} & $\begin{array}{l}\text { Arc intensity } \\
\text { and tempera- } \\
\text { ture class } \\
\mathrm{K} \text { and } \mathrm{C}\end{array}$ & $\begin{array}{l}\nu(\text { vac. }) \\
\mathrm{cm}^{-1}\end{array}$ & Zeeman effect & $\begin{array}{l}\text { Spec- } \\
\text { trum } \\
\mathrm{I}\end{array}$ \\
\hline & $\begin{array}{r}2 \\
300 \\
4 \\
100 \\
2\end{array}$ & $\begin{array}{l}(?) \\
100 \\
4 \mathrm{~h} \\
200 \\
1\end{array}$ & $\begin{array}{l}\text { 2n III(?) } \\
500 \mathrm{I} \\
4 \mathrm{III}(?) \\
100 \mathrm{III} \\
2 \mathrm{III} \\
\mathrm{A}(?)\end{array}$ & $\begin{array}{r}15,264.92 \\
278.90 \\
310.37 \\
316.77 \\
324.12\end{array}$ & $\begin{array}{l}(0.33,0.99) 0.00,0.74,1.41,2.07 \\
(0.00,0.22) 0.94 A^{2}\end{array}$ & $\begin{array}{l}\text { I } \\
\text { II } \\
\text { II }\end{array}$ \\
\hline $\begin{array}{l}20.70 \\
19.30\end{array}$ & $\begin{array}{r}\cdot 15 \\
3\end{array}$ & $\begin{array}{l}3 \\
1\end{array}$ & $20 \mathrm{IV}$ & $\begin{array}{l}331.55 \\
334.84\end{array}$ & $(0.00 \mathrm{~h}) 1.10 \mathrm{~h}$ & $\begin{array}{l}\mathrm{I} \\
\mathrm{I}\end{array}$ \\
\hline $\begin{array}{l}6,500.25 \\
6,498.19\end{array}$ & $\begin{array}{r}1 \\
30\end{array}$ & $\overline{2}$ & $\begin{array}{l}6 \mathrm{IV} \\
7 \mathrm{IV}(?) \mathrm{E}\end{array}$ & $\begin{array}{l}- \\
365.59 \\
384.66\end{array}$ & $(0.00) 0.81$ & ${ }_{\text {II }}^{1}$ \\
\hline $\begin{array}{l}92.86 \\
85.54 \mathrm{C}\end{array}$ & $\begin{array}{r}4 \\
20\end{array}$ & $\begin{array}{l}2 \\
5\end{array}$ & $\begin{array}{c}5 \mathrm{~V} \\
20 \mathrm{II} \mathrm{A}\end{array}$ & $\begin{array}{l}397.29 \\
414.66\end{array}$ & $(0.00) 1.10$ & $\mathrm{I}$ \\
\hline $\begin{array}{l}\text { 80. } 20 \\
68.9 \\
55.99\end{array}$ & $\begin{array}{r}1 \\
10 \\
250\end{array}$ & $\begin{array}{r}\overline{3} \\
100\end{array}$ & $\begin{array}{l}8 \mathrm{II} \mathrm{A} \\
300 \mathrm{I}\end{array}$ & $\begin{array}{l}426.42 \\
485.22\end{array}$ & $(0.00) 1.18$ & $\begin{array}{l}1 \\
\mathrm{I} \\
\mathrm{I}\end{array}$ \\
\hline $\begin{array}{l}54.50 \mathrm{c} \\
50.34\end{array}$ & $\begin{array}{r}150 \\
6\end{array}$ & $\begin{array}{r}50 \\
3 \\
\end{array}$ & $\begin{array}{l}200 \mathrm{I} \\
8 \mathrm{II} \mathrm{A}\end{array}$ & $\begin{array}{l}488.80 \\
498.78\end{array}$ & $\begin{array}{l}(0.38,1.11) 0.80,1.54,2.26 \\
(0.00) 1.29\end{array}$ & I \\
\hline $\begin{array}{l}48.25 \\
48.10\end{array}$ & $\begin{array}{r}4 \\
20\end{array}$ & $\begin{array}{r}2 \\
10\end{array}$ & $\begin{array}{l}10(?) \text { II A } \\
50(?) \text { II A }\end{array}$ & $\begin{array}{l}503.81 \\
504.17\end{array}$ & . & I \\
\hline $\begin{array}{l}46.62 \\
43.05 \\
26.60 \\
20.90 \\
17.23\end{array}$ & $\begin{array}{r}15 \\
3 \\
2 \\
1 \\
2\end{array}$ & $\begin{array}{c}200 \\
50 \mathrm{~h} \\
\frac{1}{1}\end{array}$ & $\begin{array}{l}5 \mathrm{~V} \mathrm{E} \\
1 \mathrm{~V}\end{array}$ & $\begin{array}{l}507.73 \\
516.32 \\
556.04 \\
569.85 \\
578.75\end{array}$ & $\begin{array}{l}(0.00 \mathrm{~W}) 1.43 \mathrm{~A}^{2} \\
(0.00 \mathrm{w}) 0.76 \mathrm{~A}^{1}\end{array}$ & $\begin{array}{c}\mathrm{II} \\
\mathrm{II} \\
\mathrm{I} \\
\mathrm{I}+\mathrm{M} \\
\mathrm{I}+\mathrm{M}\end{array}$ \\
\hline $\begin{array}{c}15.39 \\
6,410.98 \\
6,399.04 \\
94.23 \mathrm{c} \\
90.48\end{array}$ & $\begin{array}{r}1 \\
200 \\
20 \\
400 \\
100\end{array}$ & $\begin{array}{r}1 \\
60 \\
400 \\
100 \\
200\end{array}$ & $\begin{array}{l}300 \mathrm{I} \\
10 \mathrm{~V}(?) \mathrm{E} \\
600 \mathrm{I} \\
80 \mathrm{III}\end{array}$ & $\begin{array}{l}583.22 \\
593.94 \\
623.04 \\
634.79 \\
643.96\end{array}$ & $\begin{array}{l}(0.00) 1.09 \\
(0.00) 1.04 \\
(0.00 \mathrm{~W}) 1.02 \mathrm{~A}^{1} \\
(0.00) 1.03\end{array}$ & $\begin{array}{l}\text { II } \\
\text { II } \\
\text { II }\end{array}$ \\
\hline $\begin{array}{l}80.48 \\
75.50 \\
75.11 \\
74.08 \\
60.20 \mathrm{c}\end{array}$ & $\begin{array}{r}1 \\
2 \\
2 \\
3 \\
30\end{array}$ & $\frac{1}{30}$ & $\begin{array}{r}1 \mathrm{~V} \\
2 \mathrm{~V} \\
1 \mathrm{~V} \\
30 \mathrm{II}\end{array}$ & $\begin{array}{l}664.48 \\
780.72 \\
681.68 \\
684.21 \\
718.44\end{array}$ & $\begin{array}{l}(0.00 \mathrm{~W}) 1.67 \mathrm{~A}^{2} \\
(0.00) 0.89\end{array}$ & $\begin{array}{l}\text { I } \\
\text { I } \\
\text { I } \\
\text { II }\end{array}$ \\
\hline $\begin{array}{l}58.12 \\
55.38 \\
53.63 \\
39.16 \\
37.88\end{array}$ & $\begin{array}{r}20 \\
3 \\
1 \\
2 \\
2\end{array}$ & $\begin{array}{l}30 \\
\frac{1}{1} \\
3\end{array}$ & $\begin{array}{l}5 \mathrm{IV} \\
4 \mathrm{III} \mathrm{A} \\
2 \mathrm{III} \mathrm{A} \\
1 \mathrm{~V}\end{array}$ & $\begin{array}{l}723.58 \\
727.88 \\
734.69 \\
770.61 \\
773.79\end{array}$ & $(0.99) \quad 0.49,1.52$ & $\begin{array}{l}\text { II } \\
\text { I }_{\text {I }} \\
{ }_{\text {II }}\end{array}$ \\
\hline $\begin{array}{l}33.74 \\
33.24 \\
30.42 \\
25.90\end{array}$ & $\begin{array}{r}3 \\
2 \\
3 \\
100\end{array}$ & $\begin{array}{r}2 \\
1 \\
1 \\
20\end{array}$ & $\begin{array}{l}2 \text { IV(?) } \\
2 \text { III(?) } \\
2 \text { III A } \\
150 \text { I }\end{array}$ & $\begin{array}{l}784.10 \\
785.35 \\
792.38 \\
803.67\end{array}$ & $(0.63,0.99) \quad 0.25,0.63,1.01,1.38$ & $\begin{array}{l}\mathrm{I} \\
\mathrm{I}+M \\
\mathrm{I}\end{array}$ \\
\hline $20.33 \mathrm{c}$ & 100 & 200 & $80 \mathrm{III}$ & 817.44 & $\begin{array}{l}1.76 \\
(0.44) 0.87 \mathrm{~B}\end{array}$ & II \\
\hline $\begin{array}{l}18.26 \\
15.79 \\
10.91 \\
10.13 \\
08.87\end{array}$ & $\begin{array}{l}5 \\
3 \\
8 \\
8 \\
2\end{array}$ & $\begin{array}{r}2 \\
50 \\
200 \\
5 \\
?\end{array}$ & $\begin{array}{l}12 \text { III A } \\
1 \mathrm{~V} \\
4 \mathrm{~V} \mathrm{E} \\
12 \mathrm{IV} \\
2 \mathrm{III}\end{array}$ & $\begin{array}{l}822.78 \\
828.96 \\
841.20 \\
843.16 \\
846.32\end{array}$ & $\begin{array}{l}(0.95) \\
(0.27) \\
(0.10 \mathrm{~B}\end{array}$ & $\mid \begin{array}{l}\text { I } \\
\text { II } \\
\text { II } \\
\text { I }\end{array}$ \\
\hline $\begin{array}{r}08.21 \\
07.25 \\
6,305.46 \\
6,206.08 \mathrm{c} \\
93.57 \mathrm{c}\end{array}$ & $\begin{array}{r}2 \\
1 \\
10 \\
100 \\
60\end{array}$ & $\begin{array}{c}? \\
20 \mathrm{~h} \\
10 \\
300 \\
10\end{array}$ & $\begin{array}{l}3 \mathrm{III} \\
\operatorname{tr} \mathrm{IV} \\
5 \mathrm{IV} \\
50 \mathrm{IV} \mathrm{E} \\
80 \mathrm{II} \mathrm{A}\end{array}$ & $\begin{array}{l}847.98 \\
850.40 \\
854.90 \\
878.52 \\
884.85\end{array}$ & $\begin{array}{l}(0.00) 0.51 \\
(0.00 \mathrm{~W}) 1.20 \mathrm{~A}^{2} \\
(0.00) 1.33\end{array}$ & $\begin{array}{l}\text { I } \\
\text { II } \\
\text { II } \\
\text { II }\end{array}$ \\
\hline $\begin{array}{l}88.56 \\
87.73 \\
78.31 \\
73.76 \\
66.00 \mathrm{c}\end{array}$ & $\begin{array}{r}6 \\
7 \\
1 \\
4 \\
40\end{array}$ & $\begin{array}{r}3 \\
3 \\
1 \\
100 \\
20\end{array}$ & $\begin{array}{l}7 \text { III A } \\
8 \text { III A } \\
2 n \text { III(?) } \\
60 \text { III }\end{array}$ & $\begin{array}{l}897.50 \\
899.60 \\
923.46 \\
935.01 \\
954.74\end{array}$ & $\begin{array}{l}(0.00) \\
(0.00) \\
(0.06\end{array}$ & $\begin{array}{l}\mathrm{I} \\
\mathrm{I} \\
\mathrm{I} \\
\mathrm{I}\end{array}$ \\
\hline $\begin{array}{c}62.30 \\
49.92 \mathrm{c} \\
38.58 \\
36.76 \mathrm{c} \\
6,236.17\end{array}$ & $\begin{array}{r}100 \\
300 \\
12 \\
7 \\
8\end{array}$ & $\begin{array}{r}300 \\
100 \\
3 \\
2 \\
3\end{array}$ & $\begin{array}{l}\left\{\begin{array}{l}30(?) \\
50(?)\end{array}\right\} \text { III } \\
500 \mathrm{I} \\
15 \mathrm{III} \text { A } \\
10 \mathrm{III} \mathrm{A} \\
12 \mathrm{IV}\end{array}$ & $\begin{array}{r}964.17 \\
15,995.79 \\
16,024.86 \\
029.54 \\
16,031.06\end{array}$ & $\begin{array}{l}(0.00 \mathrm{w}) 1.10 \mathrm{~A}^{1} \\
(0.00) 1.13\end{array}$ & $\begin{array}{l}\text { II } \\
\text { I } \\
\text { I } \\
\text { I } \\
\text { I }\end{array}$ \\
\hline
\end{tabular}


TABLE 1.-Wave lengths and Zeeman effects in lanthanum spectra-Continued

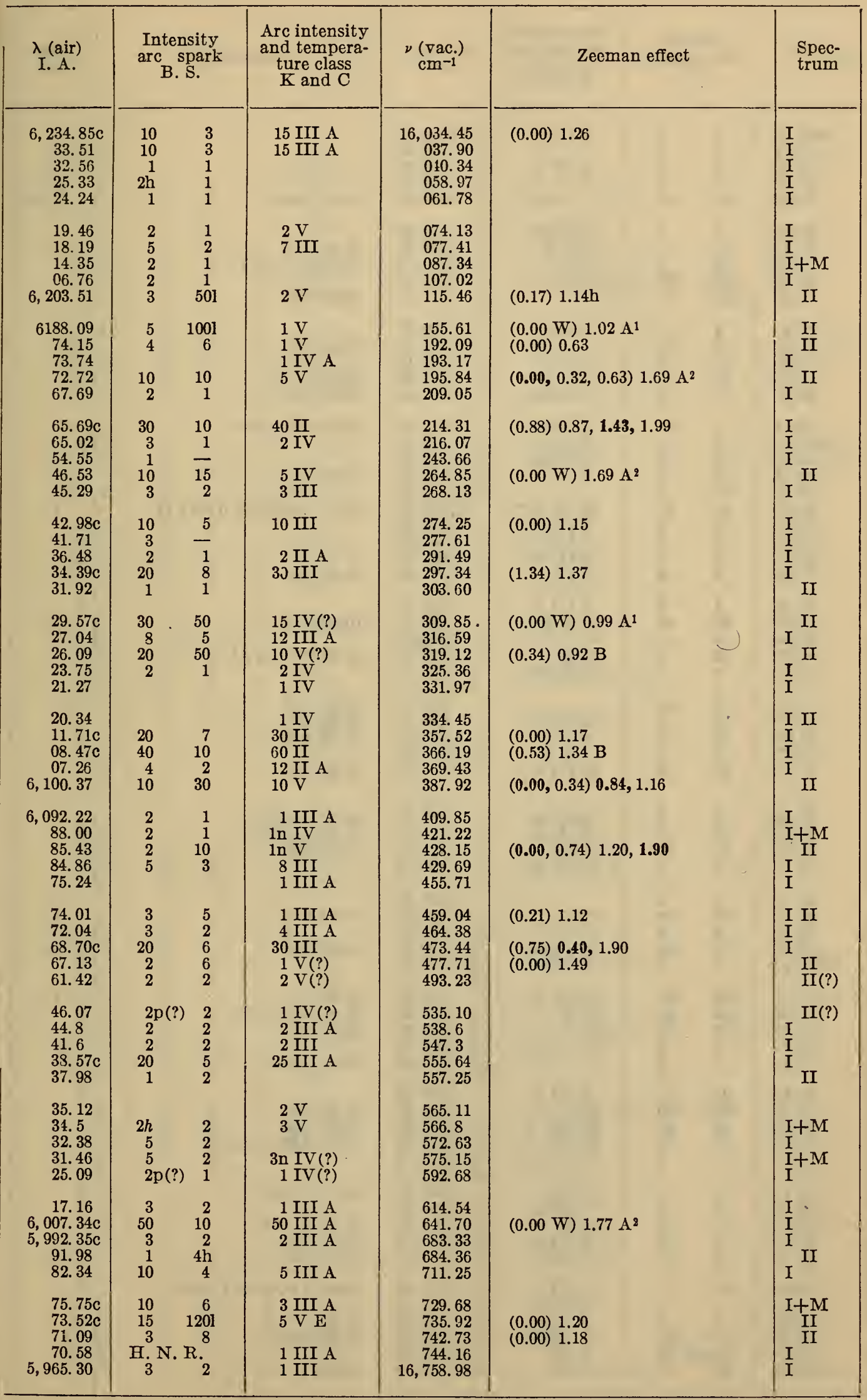


TABLE 1.-Wave lengths and Zeeman effects in lanthanum spectra-Continued

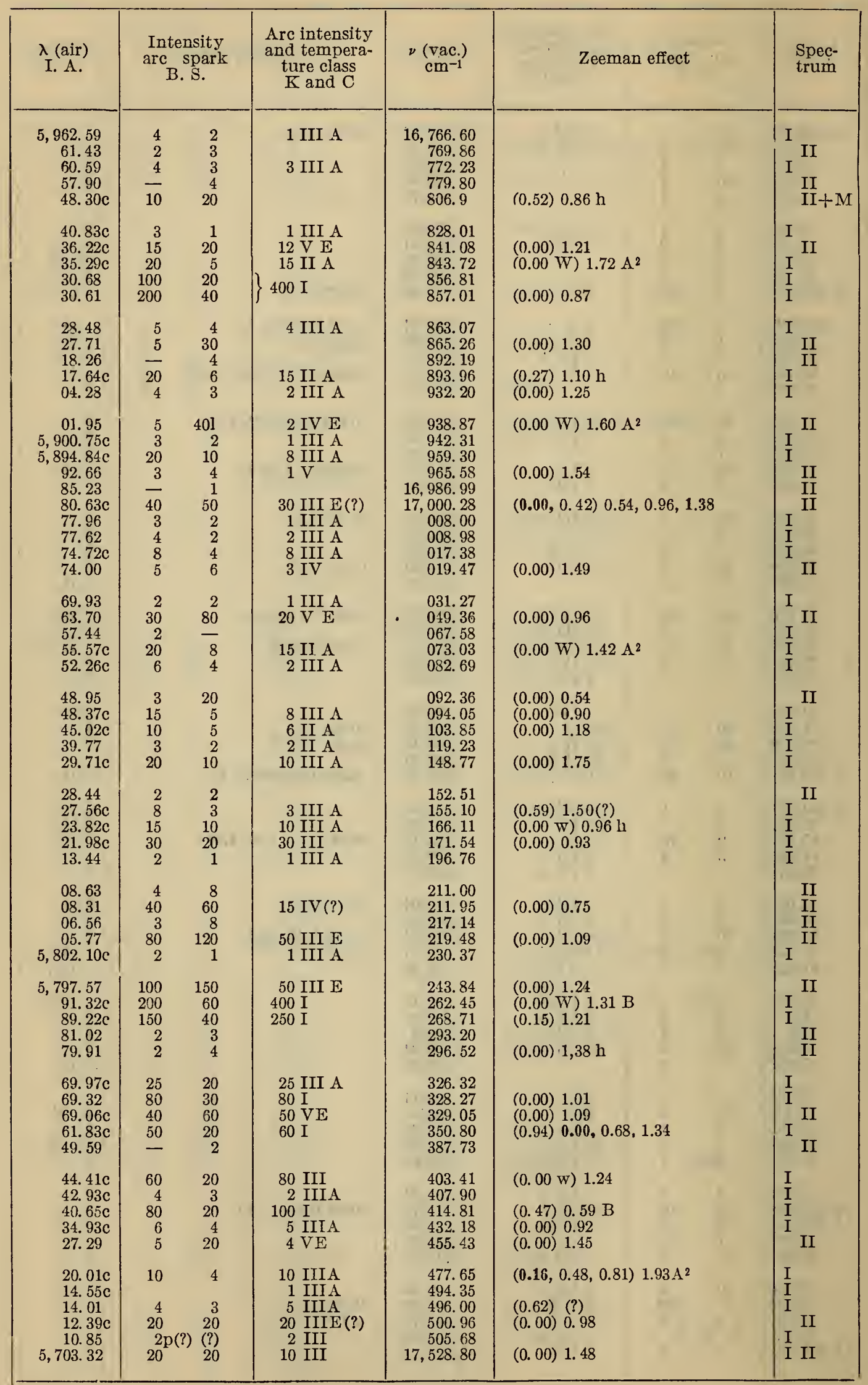


TABLE 1.-Wave lengths and Zeeman effects in lanthanum spectra-Continued

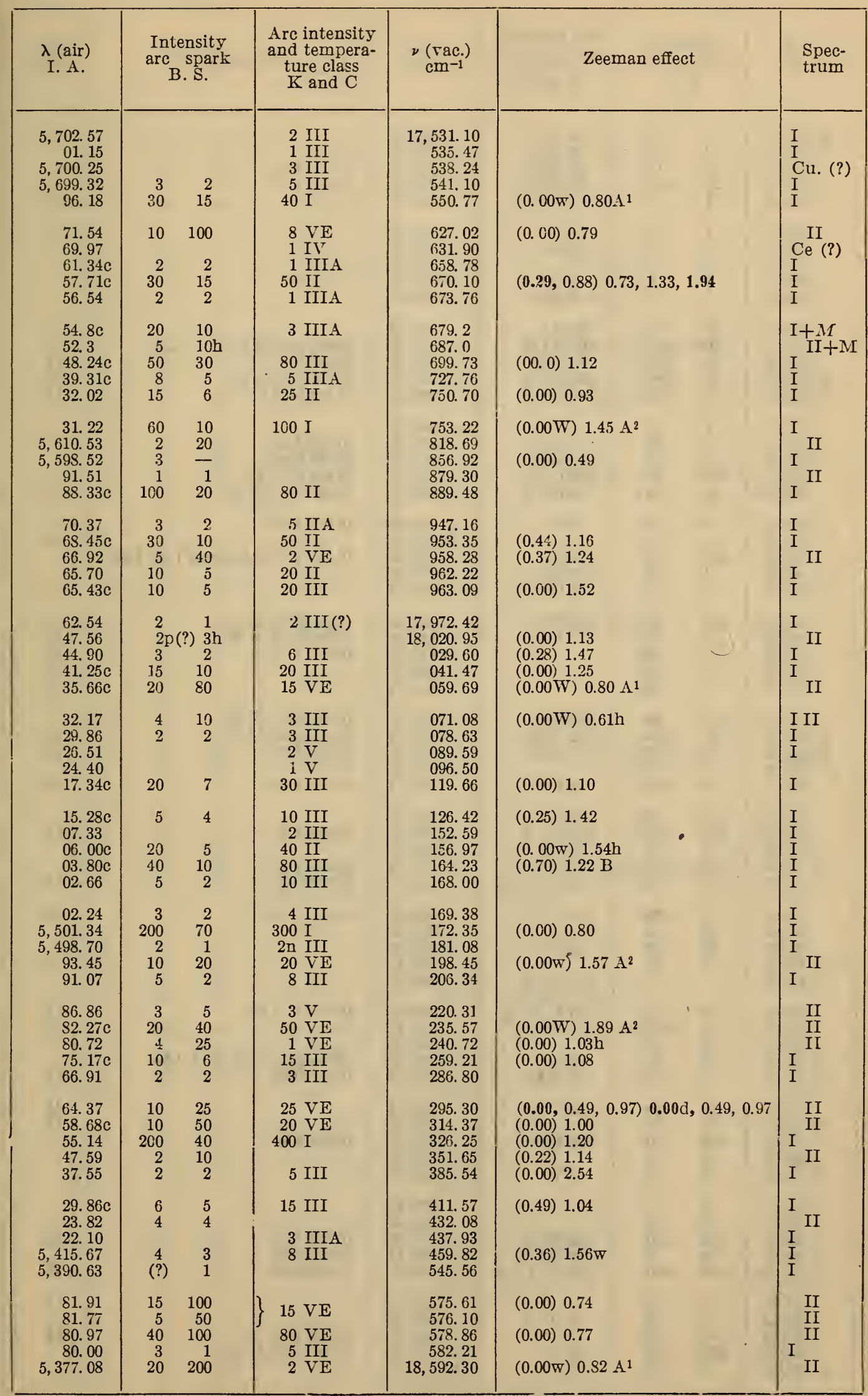


TABLE 1.-Wave lengths and Zeeman effects in lanthanum spectra-Continued

\begin{tabular}{|c|c|c|c|c|c|c|}
\hline $\begin{array}{l}\lambda \text { (air) } \\
\text { I. A. }\end{array}$ & \multicolumn{2}{|c|}{$\begin{array}{l}\text { Intensity } \\
\text { arc spark } \\
\text { B. S. }\end{array}$} & $\begin{array}{l}\text { Arc intensity } \\
\text { and tempera- } \\
\text { ture class } \\
\mathrm{K} \text { and } \mathrm{C}\end{array}$ & $\begin{array}{c}\nu\left(\mathrm{vac}^{-}\right) \\
\mathrm{cm}^{-1}\end{array}$ & Zeeman effect & $\begin{array}{l}\text { Spec- } \\
\text { trum }\end{array}$ \\
\hline $\begin{array}{l}5,300.87 \\
59.70 \\
57.85 \mathrm{c} \\
40.66 \\
33.42\end{array}$ & $\begin{array}{r}4 \\
2 \\
25 \\
20 \\
1\end{array}$ & $\begin{array}{r}2 \\
1 \\
10 \\
100 \\
2\end{array}$ & $\begin{array}{l}8 \text { III } \\
2 \text { IIIA } \\
60 \text { III } \\
40 \text { IIIE }\end{array}$ & $\begin{array}{r}18,631.14 \\
652.59 \\
659.03 \\
719.09 \\
744.50\end{array}$ & 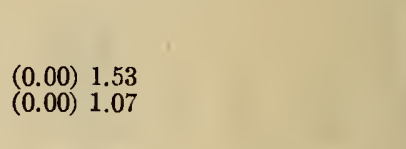 & $\begin{array}{l}\text { I } \\
\text { I }^{\text {I }} \\
\text { II } \\
\text { II }\end{array}$ \\
\hline $\begin{array}{l}30.64 \\
23.56 \\
21.34 \\
20.14 \\
07.52\end{array}$ & $\begin{array}{l}1 \\
3 \\
1 \\
3 \\
3\end{array}$ & $\begin{array}{l}2 \\
2 \\
1 \\
2 \\
2\end{array}$ & $\begin{array}{l}3 \text { III } \\
3 \text { III } \\
3 \text { III }\end{array}$ & $\begin{array}{l}754.27 \\
779.22 \\
787.04 \\
791.28 \\
835.96\end{array}$ & 4 1) & $\begin{array}{l}\text { I(?) } \\
\text { I } \\
\text { I } \\
\text { I } \\
\text { I }\end{array}$ \\
\hline $\begin{array}{c}04.01 \mathrm{c} \\
03.54 \\
02.62 \\
5,301.97 \mathrm{c} \\
5,290.83\end{array}$ & $\begin{array}{l}20 \\
50 \\
30 \\
80 \\
30\end{array}$ & $\begin{array}{r}5 \\
100 \\
150 \\
200 \\
50\end{array}$ & $\begin{array}{l}30 \text { III } \\
100 \text { III E } \\
40 \text { V E } \\
200 \text { III E } \\
60 \text { III E }\end{array}$ & $\begin{array}{l}848.43 \\
850.10 \\
853.37 \\
855.68 \\
895.38\end{array}$ & $\begin{array}{l}(0.00,0.61) 0.57,1.17,1.78 \\
(0.00) 1.16 \\
(0.00 \mathrm{w}) 1.50 \mathrm{w} \\
(0.34) 0.82 \mathrm{~B}\end{array}$ & $\begin{array}{l}\text { I } \\
\text { II } \\
\text { II } \\
\text { II } \\
\text { II }\end{array}$ \\
\hline $\begin{array}{l}87.45 \\
79.11 \\
76.40 \\
71.18 \\
59.38 \mathrm{c}\end{array}$ & $\begin{array}{r}1 \\
6 \\
5 \\
100 \\
30\end{array}$ & $\begin{array}{r}1 \\
40 \\
4 \\
30 \\
50\end{array}$ & $\begin{array}{l}1 \mathrm{~V} \\
2 \mathrm{~V} \mathrm{E} \\
10 \mathrm{III} \\
150 \mathrm{I} \\
40 \mathrm{III} \mathrm{E}\end{array}$ & $\begin{array}{r}907.46 \\
937.33 \\
947.06 \\
18,965.82 \\
19,008.37\end{array}$ & $\begin{array}{l}(0.00) 1.20 \\
(0.00) 0.90 \\
(0.00) 1.39 \mathrm{~A}^{2} \\
(0.00 \mathrm{~W}) 0.46 \mathrm{~A}^{1}\end{array}$ & 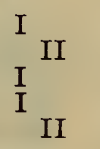 \\
\hline $\begin{array}{l}57.83 \\
57.28 \\
53.45 \\
40.81 \\
39.54\end{array}$ & $\begin{array}{r}\frac{4}{100} \\
4 \\
4\end{array}$ & $\begin{array}{r}3 \\
2 \\
30 \\
3 \\
3\end{array}$ & $\begin{array}{l}3 \text { III } \\
100 \text { I } \\
2 \text { III } \\
4 \text { III }\end{array}$ & $\begin{array}{l}013.98 \\
015.96 \\
029.83 \\
075.72 \\
080.35\end{array}$ & $\begin{array}{l}(0.00) 1.07 \\
(0.23,0.69) 0.57,1.03,1.49,1.95\end{array}$ & $\begin{array}{l}\text { I } \\
\text { I } \\
\text { I } \\
\text { I }\end{array}$ \\
\hline $\begin{array}{l}34.27 \mathrm{c} \\
26.20 \\
22.48 \\
21.32 \\
17.83\end{array}$ & $\begin{array}{r}150 \\
\frac{2}{-} \\
-\end{array}$ & $\begin{array}{c}40 \\
40 \mathrm{l} \\
3 \mathrm{~h} \\
3 \mathrm{~h} \\
10 \mathrm{~h}\end{array}$ & $\begin{array}{l}300 \mathrm{II} \\
1 \mathrm{~V} \mathrm{E}\end{array}$ & $\begin{array}{l}099.56 \\
129.05 \\
142.68 \\
146.93 \\
159.74\end{array}$ & $\begin{array}{l}(0.00 \mathrm{~W}) 2.10 \mathrm{~A}^{2} \\
(0.00 \mathrm{~W}) 0.32 \mathrm{~W} \\
(0.00) 1.15\end{array}$ & $\begin{array}{l}\text { I } \\
\text { II } \\
\text { II } \\
\text { II } \\
\text { II }\end{array}$ \\
\hline $\begin{array}{l}11.85 \mathrm{c} \\
5,204.14 \\
5,191.50 \\
90.34 \\
88.21\end{array}$ & $\begin{array}{r}150 \\
25 \\
1 \\
8 \\
40\end{array}$ & $\begin{array}{c}40 \\
300 \\
3 \mathrm{~h} \\
4 \\
500\end{array}$ & $\begin{array}{l}300 \mathrm{II} \\
12 \mathrm{~V} \mathrm{E} \\
25 \mathrm{VE}\end{array}$ & $\begin{array}{l}181.72 \\
210.14 \\
256.91 \\
261.21 \\
269.12\end{array}$ & $\begin{array}{l}(0.00 \mathrm{~W}) 1.73 \mathrm{~A}^{2} \\
(0.00) 0.86 \mathrm{~A}^{1}(?) \\
(0.00) 1.05 \\
(0.00) 1.09\end{array}$ & $\begin{array}{lll}\text { I } & \\
& \text { II } \\
& \text { II } \\
& \text { II }\end{array}$ \\
\hline $\begin{array}{l}83.91 \\
83.42 \mathrm{c} \\
79.11 \\
77.30 \mathrm{c} \\
73.83\end{array}$ & $\begin{array}{r}10 \\
200 \\
2 \\
150 \\
10\end{array}$ & $\begin{array}{c}10(?) \\
400 \\
2 \\
50 \\
251\end{array}$ & $\begin{array}{l}20 \text { II } \\
400 \text { III E } \\
2 \text { III } \\
300 \text { II } \\
10 \text { IV (?) E }\end{array}$ & $\begin{array}{l}285.10 \\
286.93 \\
302.98 \\
309.72 \\
322.67\end{array}$ & $\begin{array}{l}(0.00) 1.31 \\
(0.00) 1.07 \\
(0.00) 1.06\end{array}$ & $\begin{array}{l}\text { I } \\
\text { II }_{\text {I }} \\
{ }_{\text {II }}\end{array}$ \\
\hline $\begin{array}{l}72.89 \\
68.95 \\
67.79 \\
67.28 \\
64.03\end{array}$ & $\begin{array}{r}5 \\
2 \\
20 \\
4\end{array}$ & $\begin{array}{c}201 \\
2 \\
10 \\
10\end{array}$ & $\begin{array}{l}1 \mathrm{VE} \\
2 q \mathrm{III} \\
1 \mathrm{~V}(?)\end{array}$ & $\begin{array}{l}326.19 \\
340.92 \\
345.26 \\
347.17 \\
359.34\end{array}$ & $(0.20) 1.03$ & 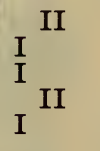 \\
\hline $\begin{array}{l}63.61 \\
62.68 \\
61.54 \\
58.68 \\
57.43\end{array}$ & $\begin{array}{r}20 \\
2 \\
2 \\
40 \\
15\end{array}$ & $\begin{array}{r}40 \\
3 \\
1 \\
20 \\
150\end{array}$ & $\begin{array}{l}20 \mathrm{~V}(?) \mathrm{E} \\
80 \mathrm{I} \\
15 \mathrm{~V} \mathrm{E}\end{array}$ & $\begin{array}{l}360.92 \\
364.41 \\
368.68 \\
379.42 \\
384.12\end{array}$ & $\begin{array}{l}(0.74) 1.18 \mathrm{~B} \\
(0.19,0.57) 1.05,1.45,1.85 \\
(0.00) 0.66\end{array}$ & $\stackrel{\text { II }}{\text { II }}^{\text {II }}$ \\
\hline $\begin{array}{l}56.74 \\
52.31 \\
45.42 \\
39.16 \\
35.42\end{array}$ & $\begin{array}{r}20 \\
1 \\
100 \\
3 \\
3\end{array}$ & $\begin{array}{r}40 \\
1 \\
40 \\
2 \\
2\end{array}$ & $\begin{array}{l}30 \mathrm{~V} \mathrm{E} \\
200 \mathrm{II} \\
2 \mathrm{~V}\end{array}$ & $\begin{array}{l}386.71 \\
403.38 \\
429.36 \\
453.03 \\
467.20\end{array}$ & $(0.00) 0.89$ & $\begin{array}{l} \\
\text { I II } \\
\text { I II } \\
\text { I } \\
\text { I }\end{array}$ \\
\hline $\begin{array}{l}34.37 \\
29.81 \\
22.99 \\
20.87 \\
14.55 \mathrm{c}\end{array}$ & $\begin{array}{r}2 \\
3 \\
100 \\
10 \\
100\end{array}$ & $\begin{array}{r}1 \\
2 \\
200 \\
4 \\
200\end{array}$ & $\begin{array}{l}150 \text { III E } \\
10 \text { III } \\
150 \text { III (?) E }\end{array}$ & $\begin{array}{l}471.18 \\
488.48 \\
514.43 \\
522.51 \\
546.63\end{array}$ & $\begin{array}{l}(0.00) 1.16 \\
(0.00) 0.92 \\
(0.00) 0.54\end{array}$ & ${ }_{\text {I II }}^{1}$ \\
\hline $\begin{array}{c}12.37 \\
09.12 \\
07.54 \\
06.23 \mathrm{c} \\
5,103.11\end{array}$ & $\begin{array}{r}\frac{1}{3} \\
\frac{1}{100} \\
3\end{array}$ & $\begin{array}{c}2 \\
2 \\
6 \mathrm{~h} \\
40 \\
2\end{array}$ & $\begin{array}{l}2 \text { III A } \\
150 \text { II } \\
2 \text { III }\end{array}$ & $\begin{array}{r}554.97 \\
567.40 \\
573.46 \\
578.48 \\
19,590.45\end{array}$ & $\begin{array}{l}(0.00) 1.19 \\
(0.19) 0.62 \mathrm{~A}^{2}(?)\end{array}$ & ${ }_{\text {I I }}^{\text {II }}$ \\
\hline
\end{tabular}


TABLE 1.-Wave lengths and Zeeman effects in lanthanum spectra-Continued

\begin{tabular}{|c|c|c|c|c|c|c|}
\hline $\begin{array}{c}\lambda \text { (air) } \\
\text { I. A. }\end{array}$ & $\begin{array}{l}\text { Int } \\
\operatorname{arc}\end{array}$ & $\begin{array}{l}\text { ensity } \\
\text { spark } \\
\text { S. }\end{array}$ & $\begin{array}{l}\text { Arc intensity } \\
\text { and tempera- } \\
\text { ture class } \\
\mathrm{K} \text { and C }\end{array}$ & $\begin{array}{l}\nu \text { (vac.) } \\
\mathrm{cm}^{-1}\end{array}$ & Zeeman effect & $\begin{array}{l}\text { Spec- } \\
\text { trum }\end{array}$ \\
\hline $\begin{array}{r}5,096.59 \\
00.56 \\
86.71 \\
86.22 \\
80.21\end{array}$ & $\begin{array}{r}2 \\
2 \\
2 \\
10\end{array}$ & $\begin{array}{c}1 \\
20 \mathrm{l} \\
3 \mathrm{hl} \\
1 \\
40\end{array}$ & $\begin{array}{l}\operatorname{liV}(?) \\
\operatorname{tr} V_{E} \\
1 \mathrm{VE}\end{array}$ & $\begin{array}{r}19,615.51 \\
638.74 \\
653.61 \\
655.50 \\
678.76\end{array}$ & $\begin{array}{l}(0.00 \mathrm{~W}) 1.33 \mathrm{~A}^{2} \\
(0.38) 0.03 \mathrm{~W}\end{array}$ & ${ }_{\text {II }}^{\text {II }}$ \\
\hline $\begin{array}{l}\text { 79. } 37 \\
78.92 \\
72.10 \\
67.90 \mathrm{c} \\
68.99\end{array}$ & $\begin{array}{r}4 \\
3 \\
3 \\
15 \\
1\end{array}$ & $\begin{array}{c}3 \\
2 \\
1 \\
10 \\
20 \mathrm{~h}\end{array}$ & $\begin{array}{l}5 \mathrm{IV} \\
2 \mathrm{~V}(?) \\
1 \mathrm{III} \mathrm{A} \\
15 \mathrm{III}\end{array}$ & $\begin{array}{l}682.01 \\
683.76 \\
710.22 \\
726.56 \\
730.10\end{array}$ & $(0.00) 1.10 \mathrm{~h}$ & $\begin{array}{l}\text { I } \\
\text { I } \\
\text { I } \\
\text { I } \\
{ }_{\text {II }}\end{array}$ \\
\hline $\begin{array}{l}63.76 \\
62.91 \\
60.85 \\
58.56 \\
56.46 \mathrm{c}\end{array}$ & $\begin{array}{r}2 \\
10 \\
2 \\
60\end{array}$ & $\begin{array}{c}3 \\
20 \\
3 \\
1 \mathrm{~h} \\
20\end{array}$ & $\begin{array}{l}4 \mathrm{~V} \mathrm{E} \\
80 \mathrm{II}\end{array}$ & $\begin{array}{l}742.68 \\
746.00 \\
754.04 \\
762.97 \\
771.18\end{array}$ & $\begin{array}{l}(0.00,0.66) 0.80,1.56,2.22 \\
(1.20) 0.00,0.79,1.57\end{array}$ & $\begin{array}{l}\text { II } \\
\text { II } \\
\text { II } \\
\text { II }\end{array}$ \\
\hline $\begin{array}{l}52.10 \\
50.57 \\
48.04 \\
46.87 \mathrm{c} \\
37.60\end{array}$ & $\begin{array}{r}1 \\
60 \\
4 \\
30 \\
2\end{array}$ & $\begin{array}{r}1 \\
20 \\
301 \\
15 \\
2\end{array}$ & $\begin{array}{l}80 \mathrm{YI} \\
1 \mathrm{VE} \\
60 \mathrm{III}\end{array}$ & $\begin{array}{l}788.25 \\
794.24 \\
804.16 \\
808.75 \\
845.20\end{array}$ & $\begin{array}{l}(0.80) 1.18 \mathrm{~B} \\
(0.00) 1.16 \\
(0.00 \mathrm{w}) 1.24\end{array}$ & $\begin{array}{l}1 \\
\text { I } \\
\text { II } \\
\text { I }\end{array}$ \\
\hline $\begin{array}{c}33.24 \\
19.50 \\
14.45 \\
02.12 \\
5,001.78 \mathrm{c}\end{array}$ & $\begin{array}{r}2 \\
10 \\
5 \\
10 \\
20\end{array}$ & $\begin{array}{c}1 \\
5 \\
30 \mathrm{hl} \\
40 \\
6\end{array}$ & $\begin{array}{l}8 \mathrm{III} \\
3 \mathrm{~V} \mathrm{E} \\
10 \mathrm{III} \mathrm{A}\end{array}$ & $\begin{array}{l}862.40 \\
916.76 \\
936.82 \\
98.59 \\
937.32\end{array}$ & $(0.00) 1.09$ & ${ }_{\text {II }}^{\text {II }}$ \\
\hline $\begin{array}{c}4,999.46 \mathrm{c} \\
96.82 \\
95.95 \\
95.17 \\
94.64\end{array}$ & $\begin{array}{r}100 \\
6 \\
1 \\
1 \\
2\end{array}$ & $\begin{array}{r}200 \\
50 \\
1 \\
1 \\
1\end{array}$ & $\begin{array}{l}200 \text { III E } \\
2 \text { V E } \\
1 \text { III A }\end{array}$ & $\begin{array}{r}19,996.60 \\
20,007.16 \\
010.64 \\
013.77 \\
015.90\end{array}$ & $(0.00 \mathrm{w}) 1.28 \mathrm{~A}^{2}$ & $\begin{array}{l}\text { II } \\
\text { II } \\
\text { II }\end{array}$ \\
\hline $\begin{array}{l}93.87 \\
91.27 \mathrm{c} \\
86.82 \mathrm{c} \\
84.92 \\
84.63\end{array}$ & $\begin{array}{r}15 \\
40 \\
60 \\
3 \\
1\end{array}$ & $\begin{array}{r}5 \\
80 \\
100 \\
2 \\
1\end{array}$ & $\begin{array}{l}20 \mathrm{II} \\
20 \mathrm{IV} \mathrm{E} \\
100 \mathrm{III} \mathrm{E} \\
2 \mathrm{IV}(?)\end{array}$ & $\begin{array}{l}018.98 \\
029.41 \\
047.28 \\
054.92 \\
056.09\end{array}$ & $\left.\begin{array}{l}(0.00) 0.85 \\
(0.00,0.43)\end{array}\right) 0.57,1.00,1.43$ & $\mathrm{I}_{\text {II }}^{\text {II }}$ \\
\hline $\begin{array}{l}83.56 \\
77.95 \\
74.20 \\
70.39 \\
68.59\end{array}$ & $\frac{2}{8}$ & $\begin{array}{c}1 \\
4 \\
4 \mathrm{~h} \\
100 \\
3\end{array}$ & $\begin{array}{c}8 \text { II A } \\
100 \text { III E } \\
2\left\{\begin{array}{c}\text { III } \\
\mathrm{V}\end{array}\right\} \quad A\end{array}$ & $\begin{array}{l}060.40 \\
083.01 \\
098.14 \\
113.55 \\
120.84\end{array}$ & $(0.00,0.29) 0.83,1.12$ us & $\begin{array}{l}\text { I } \\
\text { II } \\
\text { II }\end{array}$ \\
\hline $\begin{array}{l}64.84 \\
57.77 \\
56.04 \\
52.06 \\
49.76\end{array}$ & $\begin{array}{r}3 \\
4 \\
2 \\
10 \\
50\end{array}$ & $\begin{array}{r}2 \\
3 \\
2 \\
40 \\
20\end{array}$ & $\begin{array}{l}4 \text { III A } \\
1 \mathrm{~V} \\
5 \mathrm{~V} \mathrm{E} \\
200 \mathrm{I}\end{array}$ & $\begin{array}{l}136.03 \\
164.75 \\
171.79 \\
188.00 \\
197.38\end{array}$ & $\begin{array}{ll}(0.00) & 1.17 \\
(0.00) & 0.84\end{array}$ & ${ }_{\text {I II }}^{\text {I }}$ \\
\hline $\begin{array}{l}45.47 \mathrm{c} \\
45.84 \\
35.61 \\
34.83 \\
25.40\end{array}$ & $\begin{array}{r}20 \\
3 \\
5 \\
40 \\
3\end{array}$ & $\begin{array}{r}50 \\
2 \\
10 \\
100 \\
2\end{array}$ & $\begin{array}{l}20 \mathrm{IV}(?) \mathrm{E} \\
5 \mathrm{III} \mathrm{A} \\
2 \mathrm{~V} \mathrm{E} \\
20 \mathrm{VE} \\
2 \mathrm{IV}\end{array}$ & $\begin{array}{l}210.81 \\
213.39 \\
255.28 \\
258.48 \\
297.27\end{array}$ & $\begin{array}{l}(0.00,0.67) 1.05,1.82 \\
(0.00) 0.97\end{array}$ & $\underset{\substack{\text { II } \\
\text { II (?) }}}{\mathrm{II}}$ \\
\hline $\begin{array}{l}21.80 \\
20.98 \\
16.62 \\
11.34 \\
05.13\end{array}$ & $\begin{array}{r}200 \\
200 \\
3 \\
4 \\
4\end{array}$ & $\begin{array}{r}300 \\
300 \\
2 \\
10 \\
3\end{array}$ & $\begin{array}{c}400 \text { III E } \\
400 \text { III E } \\
1 \text { III(?) A } \\
4 \text { III A }\end{array}$ & $\begin{array}{l}312.12 \\
315.50 \\
333.52 \\
355.38 \\
381.15\end{array}$ & $\begin{array}{l}(0.00) 1.11 \\
(0.00) 1.00\end{array}$ & $\begin{array}{l}\text { II } \\
\text { II } \\
\text { II } \\
\text { II }\end{array}$ \\
\hline $\begin{array}{r}04.43 \\
4,901.87 \\
4,899.92 \\
94.24 \\
91.43\end{array}$ & $\begin{array}{r}1 \\
15 \\
150 \\
2 \\
4\end{array}$ & $\begin{array}{c}2 \mathrm{~h} \\
10 \\
200 \\
2 \\
10\end{array}$ & $\begin{array}{l}25 \text { I } \\
300 \text { III } \mathrm{E} \\
1 \text { III }\end{array}$ & $\begin{array}{l}384.05 \\
394.70 \\
402.82 \\
426.49 \\
438.23\end{array}$ & $\begin{array}{l}(0.00) \\
(0.00) \\
0.84\end{array}$ & $\mathrm{I}_{\mathrm{II}}^{\mathrm{II}}$ \\
\hline $\begin{array}{r}87.60 \\
86.82 \\
81.94 \\
80.22 \\
4,878.86\end{array}$ & $\begin{array}{r}4 \\
3 \\
1 \\
1 \\
10\end{array}$ & $\begin{array}{c}3 \\
2 \\
1 \\
10 \mathrm{~h} \\
10\end{array}$ & $\begin{array}{l}5 \mathrm{III} \\
2 \mathrm{IV}\end{array}$ & $\begin{array}{r}454.24 \\
457.51 \\
477.96 \\
485.17 \\
20,490.89\end{array}$ & $(0.00) 1.10 \mathrm{R}$ & $\begin{array}{l}\mathrm{I} \\
\mathrm{I} \\
\mathrm{I}\end{array}$ \\
\hline
\end{tabular}


TABLE 1.-Wave lengths and Zeeman effects in lanthanum spectra-Continued

\begin{tabular}{|c|c|c|c|c|c|c|c|}
\hline $\begin{array}{l}\lambda \text { (air) } \\
\text { I. A. }\end{array}$ & \multicolumn{2}{|c|}{$\begin{array}{l}\text { Intensity } \\
\text { arc spark } \\
\text { B. S. }\end{array}$} & $\begin{array}{l}\text { Arc intensity } \\
\text { and tempera- } \\
\text { ture class } \\
\mathrm{K} \text { and } \mathrm{C}\end{array}$ & $\begin{array}{l}\nu(\mathrm{vac} .) \\
\mathrm{cm}^{-1}\end{array}$ & \multicolumn{2}{|c|}{ Zeeman effect } & $\begin{array}{l}\text { Spec- } \\
\text { trum }\end{array}$ \\
\hline $\begin{array}{r}4,874.99 \\
70.56 \\
68.90 \\
67.37 \\
60.90\end{array}$ & $\begin{array}{r}1 \\
5 \\
3 \\
3 \\
60\end{array}$ & $\begin{array}{r}1 \\
2 \\
1 \\
1 \\
80\end{array}$ & $\begin{array}{l}5 \text { III } \\
2 \text { IV(?) } \\
100 \text { III E }\end{array}$ & $\begin{array}{r}20,507.15 \\
525.81 \\
532.80 \\
539.26 \\
566.59\end{array}$ & $(0.00) 1.19$ & 1 & $\begin{array}{l}\text { II } \\
I^{I} \\
\\
\text { II }\end{array}$ \\
\hline $\begin{array}{l}59.18 \\
54.95 \mathrm{c} \\
50.81 \\
50.58 \\
43.29\end{array}$ & $\begin{array}{r}2 \\
8 \\
20 \\
20 \\
4\end{array}$ & $\begin{array}{c}5 \mathrm{~h} \\
6 \\
10 \\
30 \\
5\end{array}$ & $\begin{array}{l}8 \mathrm{III} \\
20 \mathrm{I} \\
5 \mathrm{~V} \text { E } \\
1 \mathrm{~V}\end{array}$ & $\begin{array}{l}573.87 \\
591.80 \\
609.37 \\
610.35 \\
641.37\end{array}$ & $\begin{array}{l}(0.00) 1.21 \mathrm{R} \\
(0.00) 1.03\end{array}$ & & $\begin{array}{l}\text { II } \\
{ }_{\text {I }}^{\text {II }} \\
\text { II }\end{array}$ \\
\hline $\begin{array}{l}40.02 \\
39.51 \\
30.51 \\
26.87 \mathrm{c} \\
24.05\end{array}$ & $\begin{array}{r}20 \\
20 \\
5 \\
10 \\
80\end{array}$ & $\begin{array}{r}30 \\
10 \\
10 \\
20 \\
100\end{array}$ & $\begin{array}{l}10 \mathrm{~V} \mathrm{E} \\
25 \text { II } \\
1 \mathrm{~V} \mathrm{E} \\
4 \mathrm{~V} \mathrm{E} \\
80 \text { III E }\end{array}$ & $\begin{array}{l}655.32 \\
657.49 \\
695.98 \\
711.59 \\
723.70\end{array}$ & $\begin{array}{ll}(0.67) & 1.30(?) \\
(0.00) & 0.89 \\
(0.00) & 0.76 \mathrm{R} \\
(0.33) & 1.08 \mathrm{R} \\
(0.00) & 0.79\end{array}$ & v & I II \\
\hline $\begin{array}{l}17.55 \\
17.17 \\
09.00 \mathrm{c} \\
04.04 \mathrm{c} \\
4,800.24\end{array}$ & $\begin{array}{r}3 \\
10 \\
60 \\
50 \\
9\end{array}$ & $\begin{array}{r}2 \\
6 \\
100 \\
80 \\
6\end{array}$ & $\begin{array}{l}4 \mathrm{IV}(?) \\
80 \mathrm{~V} \mathrm{E} \\
50 \mathrm{~V} \mathrm{E} \\
8 \mathrm{III}\end{array}$ & $\begin{array}{l}751.66 \\
753.29 \\
788.55 \\
810.02 \\
826.49\end{array}$ & $\begin{array}{ll}(0.00) & 0.51 \\
(0.95) & 0.49,1.43\end{array}$ & & $\stackrel{\text { II }}{\text { II }}_{\text {I }}^{\text {I }}$ \\
\hline $\begin{array}{r}4,799.99 \\
96.67 \\
94.55 \\
92.46 \\
91.77\end{array}$ & $\begin{array}{r}8 \\
10 \\
3 \\
1 \\
1\end{array}$ & $\begin{array}{r}5 \\
25 \\
3 \\
1 \\
-\end{array}$ & $\begin{array}{l}8 \text { III } \\
4 \mathrm{~V} \mathrm{E} \\
2 \mathrm{~V} \mathrm{E} \\
\operatorname{tr} \text { III A }\end{array}$ & $\begin{array}{l}827.57 \\
841.99 \\
851.20 \\
860.30 \\
863.30\end{array}$ & & & ${ }_{\text {I II }}^{\text {II }}$ \\
\hline $\begin{array}{l}91.39 \\
80.55 \mathrm{c} \\
79.89 \mathrm{c} \\
75.14 \mathrm{c} \\
70.43\end{array}$ & $\begin{array}{r}5 \\
2 \\
4 \\
3 \\
10\end{array}$ & $\begin{array}{l}3 \\
2 \\
3 \\
3 \\
8\end{array}$ & $\begin{array}{l}5 \mathrm{II} \\
1 \mathrm{~V} \\
4 \mathrm{II} \\
2 \mathrm{~V} \\
15 \mathrm{II}\end{array}$ & $\begin{array}{l}864.96 \\
912.27 \\
915.16 \\
935.96 \\
956.63\end{array}$ & $(0.00) 0.93 \mathrm{R}$ & n & $\begin{array}{l}\text { I } I(?(?) \\
\text { I } \\
\text { I(?) } \\
\text { I }\end{array}$ \\
\hline $\begin{array}{l}67.80 \\
66.89 \\
59.71 \mathrm{c} \\
58.40 \\
57.14 \mathrm{c}\end{array}$ & $\begin{array}{c}1 \mathrm{~h} \\
60 \\
2 \\
2 \\
3\end{array}$ & $\begin{array}{c}1 \mathrm{~h} \\
30 \\
2 \\
3 \\
2\end{array}$ & $\begin{array}{l}100 \mathrm{I} \\
2 \mathrm{IV}(?) \\
2 \mathrm{IV}\end{array}$ & $\begin{array}{r}968.19 \\
20,972.19 \\
21,003.83 \\
009.61 \\
015.18\end{array}$ & $(0.00) 0.99$ & & $\begin{array}{l}\mathrm{I} \\
\mathrm{I} \\
\mathrm{I}\end{array}$ \\
\hline $\begin{array}{l}56.97 \\
53.11 \mathrm{c} \\
52.41 \mathrm{c} \\
50.41 \mathrm{c} \\
48.73\end{array}$ & $\begin{array}{r}2 \\
3 \\
10 \\
80\end{array}$ & $\begin{array}{r}1 \\
2 \\
8 \\
150\end{array}$ & $\begin{array}{c}1 \mathrm{~V} \\
1 \mathrm{IV} \\
3 \text { III } \\
15 \text { III } \\
100 \mathrm{~V} \mathrm{E}\end{array}$ & $\begin{array}{l}015.93 \\
032.99 \\
036.09 \\
044.95 \\
052.39\end{array}$ & $\begin{array}{l}(0.00) 1.04 \mathrm{R} \\
(0.00) 1.00\end{array}$ & $r=$ & $\begin{array}{l}\text { I } \\
\text { I } \\
I \\
I_{\text {II }}\end{array}$ \\
\hline $\begin{array}{l}43.08 \mathrm{c} \\
40.27 \\
39.80 \\
33.82 \mathrm{c} \\
30.73\end{array}$ & $\begin{array}{r}100 \\
100 \\
6 \\
8 \\
2\end{array}$ & $\begin{array}{c}250 \\
120 \\
15 \\
5 \\
3 \mathrm{~h}\end{array}$ & $\begin{array}{l}100 \mathrm{~V} \mathrm{E} \\
150 \text { III E } \\
2 \mathrm{~V} \mathrm{E} \\
4 \mathrm{~V}(?)\end{array}$ & $\begin{array}{l}077.47 \\
089.96 \\
092.06 \\
118.70 \\
132.50\end{array}$ & $\begin{array}{l}(0.52 \mathrm{w}) 0.95 \mathrm{~B} \\
(0.00 \mathrm{w}) 0.97 \mathrm{~A}^{1}\end{array}$ & & $\mathrm{I}_{\text {II }}^{\text {II }}$ \\
\hline $\begin{array}{l}29.09 \\
28.41 \mathrm{c} \\
24.42 \\
23.72 \\
22.14\end{array}$ & $\begin{array}{r}1 \\
80 \\
20 \\
2 \\
1\end{array}$ & $\begin{array}{c}\overline{100} \\
40 \\
1 \\
2 \mathrm{~h}\end{array}$ & $\begin{array}{l}1 \mathrm{~V} \\
80 \mathrm{~V}(?) \mathrm{E} \\
6 \mathrm{VE} \\
3 \mathrm{II}\end{array}$ & $\begin{array}{l}139.82 \\
142.86 \\
160.72 \\
163.85 \\
170.94\end{array}$ & $\begin{array}{l}(0.00,0.32) 1.31,1 \\
(0.65) 0.95 \text { us }\end{array}$ & $.61,1.91$ & $\begin{array}{l}\text { I(?) } \\
\text { II } \\
\text { II } \\
\text { I } \\
\text { II }\end{array}$ \\
\hline $\begin{array}{l}19.93 \mathrm{c} \\
17.58 \\
16.44 \mathrm{c} \\
14.14 \\
12.92 \mathrm{c}\end{array}$ & $\begin{array}{r}40 \\
10 \\
40 \\
4 \\
20\end{array}$ & $\begin{array}{r}150 \\
50 \\
80 \\
2 \\
40\end{array}$ & $\begin{array}{r}15 \mathrm{~V} \text { E } \\
4 \mathrm{~V} \text { E } \\
25 \mathrm{~V} \text { E } \\
5 \mathrm{I} \\
12 \mathrm{~V} \text { E }\end{array}$ & $\begin{array}{l}180.85 \\
191.40 \\
196.52 \\
206.86 \\
212.35\end{array}$ & 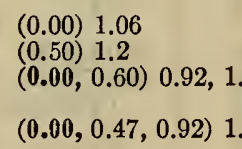 & $\begin{array}{l}.52,2.12 \\
.83,2.26 \mathrm{R}\end{array}$ & $\begin{array}{l}\text { II } \\
\text { II } \\
\text { II } \\
\text { II }\end{array}$ \\
\hline $\begin{array}{r}08.18 \mathrm{c} \\
03.27 \mathrm{c} \\
02.64 \\
4,700.26 \mathrm{c} \\
4,699.62 \mathrm{c}\end{array}$ & $\begin{array}{r}8 \\
30 \\
8 \\
8 \\
20\end{array}$ & $\begin{array}{r}8 \\
150 \\
6 \\
8 \\
50\end{array}$ & $\begin{array}{l}8 \mathrm{III}(?) \\
20 \mathrm{VE} \\
10 \mathrm{I} \\
8 \mathrm{III}(?) \\
20 \mathrm{VE}\end{array}$ & $\begin{array}{l}233.71 \\
255.87 \\
258.72 \\
269.48 \\
272.38\end{array}$ & $\begin{array}{l}(0.00) 0.97 \mathrm{R} \\
(0.00 \mathrm{~W}) 1.70 \mathrm{~A}^{2}\end{array}$ & & $\underbrace{\text { I }}_{\text {I II }}$ \\
\hline $\begin{array}{r}95.30 \\
92.50 \mathrm{c} \\
91.17 \mathrm{c} \\
88.65 \\
4,634.39\end{array}$ & $\begin{array}{r}3 \\
50 \\
25 \\
8 \\
1\end{array}$ & $\begin{array}{c}3 \\
200 \\
50 \\
40 \\
2 \mathrm{~h}\end{array}$ & $\begin{aligned} 50 \mathrm{~V} \mathrm{E} \\
30 \mathrm{~V} \\
2 \mathrm{~V} \mathrm{E}\end{aligned}$ & $\begin{array}{r}291.95 \\
304.66 \\
310.70 \\
322.15 \\
21,341.54\end{array}$ & $\begin{array}{l}(0.00 \mathrm{~W}) 1.21 \mathrm{~A}^{2} \\
(0.00,0.46) 0.46 \text { us } \\
(0.00) 0.96\end{array}$ & $s$ & $\begin{array}{l}\text { I } \\
\text { II } \\
\text { II } \\
\text { II } \\
\text { II }\end{array}$ \\
\hline
\end{tabular}


TABLE 1.-Wave lengths and Zeeman effects in lanthanum spectra-Continued

\begin{tabular}{|c|c|c|c|c|c|c|}
\hline \multirow{3}{*}{$\begin{array}{c}\lambda \text { (air) } \\
\text { I. A. } \\
4,682.12 \\
73.53 \\
71.82 \mathrm{c} \\
68.91 \mathrm{c}\end{array}$} & \multicolumn{2}{|c|}{$\begin{array}{l}\text { Intensity } \\
\text { arc spark } \\
\text { B. S. }\end{array}$} & \multirow{2}{*}{$\begin{array}{c}\text { Arc intensity } \\
\text { and tempera- } \\
\text { ture class } \\
\mathrm{K} \text { and } \mathrm{C}\end{array}$} & \multirow{3}{*}{$\begin{array}{r}\nu \text { (vac.) } \\
\mathrm{Cm}^{-1} \\
\\
21,351.89 \\
391.14 \\
398.96 \\
412.30\end{array}$} & Zeeman effect & $\begin{array}{l}\text { Spec- } \\
\text { trum }\end{array}$ \\
\hline & 4 & 5 & & & & II \\
\hline & $\begin{array}{l}30 \\
40\end{array}$ & $\begin{array}{l}200 \\
250\end{array}$ & $\begin{array}{l}40 \mathrm{~V} \mathrm{E} \\
50 \mathrm{VE}\end{array}$ & & $\begin{array}{l}(0.00 \mathrm{w}) 1.29 \mathrm{~h} \\
(0.000 .34,0.68) 0.77,1.12,1.47 \text {, }\end{array}$ & II \\
\hline $63.76 \mathrm{c}$ & 50 & 300 & $50 \mathrm{~V} \mathrm{E}$ & 435.94 & $\begin{array}{l}1.81 \text { us } \\
(0.20) 0.99\end{array}$ & II \\
\hline $\begin{array}{l}62.51 \\
60.70 \\
55.49 \mathrm{c} \\
53.90 \mathrm{c} \\
52.07\end{array}$ & $\begin{array}{r}100 \\
8 \\
80 \\
4 \\
15\end{array}$ & $\begin{array}{r}200 \\
8 \\
400 \\
3 \\
30 \mathrm{hl}\end{array}$ & $\begin{array}{l}200 \text { III E } \\
8 \text { III } \\
150 \mathrm{~V} \mathrm{E} \\
20 \mathrm{I}\end{array}$ & $\begin{array}{l}441.69 \\
450.02 \\
474.02 \\
481.36 \\
489.81\end{array}$ & $\begin{array}{l}(0.00 \mathrm{~W}) 0.89 \mathrm{~A}^{2} \\
(0.00) 0.92 \mathrm{R} \\
(0.43) 1.20 \mathrm{~B}\end{array}$ & $\begin{array}{l}\text { II } \\
\text { II } \\
\text { I II(?) }\end{array}$ \\
\hline $\begin{array}{l}50.32 \\
48.64 \\
47.50 \mathrm{c} \\
46.33 \\
45.28\end{array}$ & $\begin{array}{l}12 \\
30 \\
25 \\
10 \\
40\end{array}$ & $\begin{array}{r}8 \\
20 \\
100 \\
10 \\
100\end{array}$ & $\begin{array}{l}15 \mathrm{I} \\
40 \mathrm{I} \\
8 \mathrm{~V} \mathrm{E} \\
12 \mathrm{III} \\
40 \mathrm{~V} \mathrm{E}\end{array}$ & $\begin{array}{l}497.90 \\
505.67 \\
510.94 \\
516.36 \\
521.22\end{array}$ & $\begin{array}{l}(0.30) 1.13 \mathrm{R} \\
(0.48) ? \\
(0.60,0.31,0.61) 1.98 \mathrm{~A}^{2} \\
(0.00) 0.89 \mathrm{R} \\
(0.60) 1.26 \mathrm{~B}\end{array}$ & ${ }_{\text {I II }}^{\text {I }}$ \\
\hline 43. $11 \mathrm{c}$ & 5 & 5 & 5 III & 531.28 & & I \\
\hline $\begin{array}{l}41.40 \\
36.42 \\
34.95 \\
33.4\end{array}$ & $\begin{array}{r}\overline{10} \\
2 \\
1\end{array}$ & $\begin{array}{l}20 \\
251 \\
10 \mathrm{hl}\end{array}$ & $4 \mathrm{VE}$ & $\begin{array}{l}539.21 \\
562.35 \\
569.19 \\
576.4\end{array}$ & $\begin{array}{ll}(0.21) & 1.00 \\
(0.00) & 1.43\end{array}$ & $\begin{array}{l}\text { II } \\
\text { II } \\
\text { II }\end{array}$ \\
\hline $\begin{array}{l}27.35 \\
23.99 \\
19.87 \mathrm{c} \\
15.06 \mathrm{c} \\
13.38\end{array}$ & $\begin{array}{r}2 \\
1 \\
100 \\
8 \\
100\end{array}$ & $\begin{array}{c}2 \\
2 \mathrm{~h} \\
300 \\
7 \\
200\end{array}$ & $\begin{array}{l}150 \text { V E } \\
15 \text { III } \\
200 \text { V E }\end{array}$ & $\begin{array}{l}604.61 \\
620.31 \\
639.59 \\
662.14 \\
670.03\end{array}$ & $\begin{array}{l}(0.00) \quad 0.70 \\
(1.31) 1.37 \mathrm{R} \\
(0.00,0.33) 0.83,1.16,1.49\end{array}$ & $\begin{array}{l}\text { I } \\
\text { II } \\
\text { II } \\
\text { II }\end{array}$ \\
\hline $\begin{array}{l}05.78 \\
05.08 \\
04.24 \\
02.04 \mathrm{c} \\
01.65\end{array}$ & $\begin{array}{r}50 \\
6 \\
6 \\
10 \\
-\end{array}$ & $\begin{array}{r}100 \\
3 \\
4 \\
10 \\
3\end{array}$ & $\begin{array}{l}50 \mathrm{~V} \text { E } \\
10 \text { III } \\
10 \text { III } \\
20 \text { III }\end{array}$ & $\begin{array}{l}705.79 \\
709.09 \\
713.05 \\
723.43 \\
725.27\end{array}$ & (0.63) $0.89,1.52$ & $\begin{array}{l}\text { II } \\
I^{\text {I }} \\
\text { I } \\
\end{array}$ \\
\hline $\begin{array}{r}4,600.59 \\
4,596.19 \\
95.06 \\
89.89 \\
87.14\end{array}$ & $\begin{array}{l}1 \\
6 \\
1 \\
5 \\
-\end{array}$ & $\begin{array}{l}5 \mathrm{~h} \\
6 \\
2 \mathrm{~h} \\
5 \\
2 \mathrm{~h}\end{array}$ & $10 \mathrm{I}$ & $\begin{array}{l}730.27 \\
751.08 \\
756.42 \\
780.93 \\
793.99\end{array}$ & 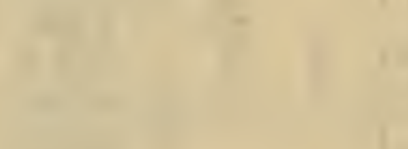 & ${ }_{\text {II }}^{\text {II }}$ \\
\hline $\begin{array}{l}81.20 \mathrm{c} \\
80.05 \\
74.87 \mathrm{c} \\
70.97 \\
70.02\end{array}$ & $\begin{array}{r}10 \\
50 \\
150 \\
6 \\
60\end{array}$ & $\begin{array}{r}10 \\
150 \\
200 \\
10 \\
50\end{array}$ & $\begin{array}{l}12 \text { III } \\
100 \text { V E } \\
300 \text { III E } \\
8 \text { V E } \\
250 \text { I }\end{array}$ & $\begin{array}{l}822.25 \\
827.73 \\
852.44 \\
871.08 \\
875.63\end{array}$ & $\begin{array}{l}(0.00) 1.51 \\
(0.49,0.98) 0.49,0.98,1.46,1.94 \\
(0.43) 1.00 \mathrm{R} \\
(0.00) 1.34\end{array}$ & $\begin{array}{l}\text { I } \\
\text { II } \\
\text { II } \\
\text { II }\end{array}$ \\
\hline $\begin{array}{l}67.90 \\
64.85 \mathrm{c} \\
62.5 \\
59.28 \\
58.46\end{array}$ & $\begin{array}{r}50 \\
6 \\
1 \\
30 \\
100\end{array}$ & $\begin{array}{c}40 \\
4 \\
5 \mathrm{~h} \\
100 \\
200\end{array}$ & $\begin{array}{l}200 \text { I } \\
12 \text { III } \\
50 \text { V E } \\
250 \text { III E }\end{array}$ & $\begin{array}{l}885.78 \\
900.41 \\
911.7 \\
927.16 \\
931.10\end{array}$ & $\begin{array}{l}(0.00) 1.24 \\
(0.00) 1.42 \mathrm{R} \\
(0.00,0.18) 1.62 \\
(0.00 \mathrm{~W}) 0.88 \mathrm{~A}^{1}\end{array}$ & $\begin{array}{l}\text { I } \\
\text { I } \\
\text { II } \\
\text { II } \\
\text { II }\end{array}$ \\
\hline $\begin{array}{l}52.47 \\
50.76 \mathrm{c} \\
50.16 \\
49.50 \\
41.78 \mathrm{c}\end{array}$ & $\begin{array}{r}8 \\
8 \\
4 \\
40 \\
10\end{array}$ & $\begin{array}{r}8 \\
6 \\
3 \\
30 \\
8\end{array}$ & $\begin{array}{l}8 \text { II A } \\
10 \text { III A } \\
5 \text { III A } \\
50 \text { I } \\
15 \text { III }\end{array}$ & $\begin{array}{r}959.96 \\
968.21 \\
971.11 \\
21,974.30 \\
22,011.65\end{array}$ & $\begin{array}{l}(0.00) 0.87 \\
(0.78) 0.38 \mathrm{R} \\
(0.00) 1.08\end{array}$ & $\begin{array}{l}\mathrm{I} \\
\mathrm{I}_{\mathrm{I}} \\
\mathrm{I} \\
\mathrm{I}\end{array}$ \\
\hline $\begin{array}{l}40.71 \\
38.87 \\
37.57 \\
30.54 \\
28.88\end{array}$ & $\begin{array}{l}3 \\
1 \\
2 \\
4 \\
3\end{array}$ & $\begin{array}{l}10 \\
8 \mathrm{hl} \\
2 \\
15 \\
2\end{array}$ & $2 \mathrm{VE}$ & $\begin{array}{l}016.84 \\
025.76 \\
032.07 \\
066.26 \\
074.34\end{array}$ & $(0.00) 1.27$ & ${ }_{\text {I II }}^{\text {II }}$ \\
\hline $\begin{array}{l}26.12 \\
25.31 \mathrm{c} \\
22.37 \\
16.38 \\
08.48\end{array}$ & $\begin{array}{r}100 \\
40 \\
200 \\
2 \\
3\end{array}$ & $\begin{array}{l}200 \\
100 \\
400 \\
5 \mathrm{hl} \\
10\end{array}$ & $\begin{array}{l}200 \text { III E } \\
20 \text { V E } \\
500 \text { III E }\end{array}$ & $\begin{array}{l}087.81 \\
091.76 \\
106.12 \\
135.44 \\
174.22\end{array}$ & $\begin{array}{l}(0.00 \mathrm{~W}) 1.01 \mathrm{~A}^{1} \\
(0.46) 1.19 \mathrm{~B} \\
(0.00) 1.02 \\
(0.00) 0.90\end{array}$ & $\begin{array}{l}\text { II } \\
\text { II } \\
\text { II } \\
\text { II } \\
\text { II }\end{array}$ \\
\hline $\begin{aligned} & 07.4 \\
& 05.82 \\
& 02.16 \\
& 01.57 \\
& 4,500.21\end{aligned}$ & $\begin{array}{r}2 \\
1 \\
1 \\
6 \\
30\end{array}$ & $\begin{array}{c}2 \\
3 \mathrm{hl} \\
10 \mathrm{hl} \\
4 \\
30\end{array}$ & $\begin{array}{l}10 \text { II A } \\
40 \text { II }\end{array}$ & $\begin{aligned} & 179.5 \\
& 187.32 \\
& 205.35 \\
& 208.26 \\
& 22,214.97\end{aligned}$ & $\begin{array}{l}(0.00) 1.47 \\
(0.00 \mathrm{~W}) 1.10 \mathrm{~A}^{1}\end{array}$ & $\mathrm{I}_{\mathrm{I}}^{\mathrm{I}}$ \\
\hline
\end{tabular}


TABLE 1.-Wave lengths and Zeeman effects in lanthanum spectra-Continued

\begin{tabular}{|c|c|c|c|c|c|c|}
\hline $\begin{array}{l}\lambda \text { (air) } \\
\text { I. A. }\end{array}$ & \multicolumn{2}{|c|}{$\begin{array}{l}\text { Intensity } \\
\text { arc spark } \\
\text { B. S. }\end{array}$} & $\begin{array}{l}\text { Arc intensity } \\
\text { and tempera- } \\
\text { cure class } \\
\mathrm{K} \text { and } \mathrm{C}\end{array}$ & $\begin{array}{l}\nu(\mathrm{vac} .) \\
\mathrm{cm}^{-1}\end{array}$ & Zecman effect & $\begin{array}{l}\text { Spec- } \\
\text { trum }\end{array}$ \\
\hline $\begin{array}{c}4,499.04 \mathrm{c} \\
98.76 \\
97.00 \\
94.71 \mathrm{c} \\
93.81\end{array}$ & $\begin{array}{r}10 \\
2 \\
2 \\
20 \\
5\end{array}$ & $\begin{array}{r}10 \\
10 \\
2 \\
15 \\
3\end{array}$ & $\begin{array}{l}10 \mathrm{III} \\
30 \mathrm{I} \\
10 \mathrm{IA}\end{array}$ & $\begin{array}{r}22,220.75 \\
222.13 \\
230.83 \\
242.16 \\
246.61\end{array}$ & 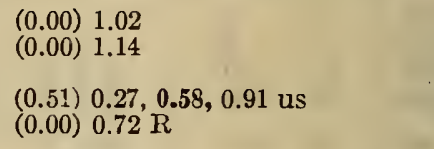 & $\begin{array}{l}\text { I } \\
\text { II } \\
\text { II }\end{array}$ \\
\hline $\begin{array}{l}93.11 \\
91.76 \mathrm{c} \\
86.05 \\
84.48 \\
81.21\end{array}$ & $\begin{array}{l}15 \\
10 \\
10 \\
3\end{array}$ & $\begin{array}{c}10 \\
8 \\
10 \\
1 \mathrm{~h} \\
25 \mathrm{~h} 1\end{array}$ & $\begin{array}{l}25 \text { I } \\
15 \text { III } \\
20 \text { III }\end{array}$ & $\begin{array}{l}250.08 \\
256.76 \\
285.04 \\
292.90 \\
309.16\end{array}$ & $\begin{array}{l}(0.00) 1.16 \\
(0.00) 1.52 \\
(0.40) 0.50,0.87\end{array}$ & $\begin{array}{l}\text { I } \\
\text { I } \\
\text { III } \\
\text { II }\end{array}$ \\
\hline $\begin{array}{l}79.82 \mathrm{c} \\
74.54 \\
74.03 \\
68.97 \\
59.10\end{array}$ & $\begin{array}{r}6 \\
4 \\
2 \\
10 \\
-\end{array}$ & $\begin{array}{r}5 \\
3 \\
10 \\
6 \\
3\end{array}$ & $\begin{array}{l}15 \text { II A } \\
5 \text { III A } \\
25 \text { II }\end{array}$ & $\begin{array}{l}316.08 \\
342.42 \\
344.96 \\
370.26 \\
419.78\end{array}$ & $(0.00,0.66) 0.50,1.17,1.83$ & ${ }_{\text {I II }}^{\text {I }}$ \\
\hline $\begin{array}{l}55.79 \\
55.21 \\
53.85 \\
52.15 \\
45.12\end{array}$ & $\begin{array}{r}20 \\
3 \\
15 \\
2\end{array}$ & $\begin{array}{r}50 \\
2 \\
15 \\
1\end{array}$ & $\begin{array}{l}25 \text { V E } \\
10 \text { II A } \\
2 \text { IV A } \\
30 \text { II } \\
2 \text { III A }\end{array}$ & $\begin{array}{l}436.43 \\
439.35 \\
446.20 \\
449.78 \\
490.79\end{array}$ & $\begin{array}{l}(0.24) 1.41 \\
(0.00) 0.98\end{array}$ & $\begin{array}{l}\text { II } \\
\text { I } \\
\text { I } \\
\text { I }\end{array}$ \\
\hline $\begin{array}{l}43.94 \mathrm{c} \\
42.68 \\
35.84 \\
32.95 \\
29.90 \mathrm{c}\end{array}$ & $\begin{array}{c}5 \\
6 \\
6 \\
10(?) \\
200\end{array}$ & $\begin{array}{l}20 \mathrm{hl} \\
3 \\
10 \\
20(?) \\
400\end{array}$ & $\begin{array}{l}10 \mathrm{I} \\
12 \mathrm{II} \\
6 \mathrm{IV} \mathrm{E} \\
500 \mathrm{III} \mathrm{E}\end{array}$ & $\begin{array}{l}496.26 \\
502.64 \\
537.34 \\
552.03 \\
567.56\end{array}$ & $\begin{array}{l}(0.00) 1.18 \mathrm{~h} \\
(0.00,0.60,1.19) 1.28,1.86,2.44 \\
(0.00,0.40) 0.51,0.91,1.31\end{array}$ & $\begin{array}{ll}\text { I } & \text { II? } \\
\text { I } & \text { II } \\
\text { II }+M\end{array}$ \\
\hline $\begin{array}{l}27.52 \mathrm{c} \\
23.90 \\
19.16 \\
17.14 \\
13.45\end{array}$ & $\begin{array}{r}30 \\
20 \\
20 \\
2\end{array}$ & $\begin{array}{c}100 \\
15 \\
30 \\
2 \mathrm{~h}\end{array}$ & $\begin{array}{l}40 \mathrm{~V} \mathrm{E} \\
30 \text { II } \\
6 \text { V E } \\
6 \mathrm{n} \mathrm{III} \\
2 \text { III A }\end{array}$ & $\begin{array}{l}579.69 \\
598.16 \\
622.41 \\
632.75 \\
651.67\end{array}$ & $\begin{array}{l}(0.00 \mathrm{w}) 1.06 \\
(0.00) 1.09 \\
(0.28) 1.04\end{array}$ & $\begin{array}{l}\text { II } \\
\text { II } \\
\text { I II }\end{array}$ \\
\hline $\begin{array}{c}12.22 \\
11.21 \\
03.02 \mathrm{c} \\
4,402.64 \\
4,397.04\end{array}$ & $\begin{array}{l}\overrightarrow{1} \\
2 \\
5\end{array}$ & $\begin{array}{l}2 \mathrm{~h} \\
25 \mathrm{hl} \\
2 \\
4\end{array}$ & $\begin{array}{l}2 \mathrm{~V} \mathrm{E} \\
8 \text { III A; } \\
15 \text { III } \\
2 \text { IV A }\end{array}$ & $\begin{array}{l}657.98 \\
663.17 \\
705.33 \\
707.29 \\
736.21\end{array}$ & $\begin{array}{l}(0.00 \mathrm{~h}) 1.13 \mathrm{~h} \\
(0.00) 0.86\end{array}$ & $\begin{array}{l}\text { II } \\
\text { II } \\
\text { II } \\
\text { I } \\
\text { I }\end{array}$ \\
\hline $\begin{array}{l}95.79 \mathrm{c} \\
96.31 \\
93.52 \mathrm{c} \\
89.87 \\
85.20 \mathrm{c}\end{array}$ & $\begin{array}{r}2 \\
6 \\
10\end{array}$ & $\begin{array}{r}1 \\
4 \\
40\end{array}$ & $\begin{array}{l}4 \text { IV A } \\
2 \text { IV } \\
4 \text { III } \\
15 \text { III } \\
20 \text { V E }\end{array}$ & $\begin{array}{l}737.50 \\
739.98 \\
754.42 \\
773.34 \\
797.59\end{array}$ & $\begin{array}{l}(0.00) 1.07 \\
(0.00) 1.24\end{array}$ & $\begin{array}{l}\text { I } \\
\text { I } \\
\text { I } \\
\text { I }_{\text {II }}\end{array}$ \\
\hline 83.44 & $20(?)$ & 100 & $25 \mathrm{~V} \mathrm{E}$ & 806.75 & $\begin{array}{l}(0.00,0.40,0.80) 0.28,0.68,1.08 \\
\quad 1.48,1,88\end{array}$ & $\mathrm{II}+\mathrm{M}$ \\
\hline $\begin{array}{l}80.55 \\
78.10 \mathrm{c} \\
64.66 \mathrm{c} \\
63.05\end{array}$ & $\begin{array}{r}4 \\
15 \\
25 \\
5\end{array}$ & $\begin{array}{r}2 \\
50 \\
100 \\
501\end{array}$ & $\begin{array}{l}12 \text { II A } \\
15 \text { IV E } \\
30 \text { IV F } \\
4 \text { V E }\end{array}$ & $\begin{array}{l}821.79 \\
834.56 \\
904.88 \\
913.33\end{array}$ & $\begin{array}{l}(0.30 \mathrm{w}) 0.95 \\
(0.00) 1.28 \\
(00.0 \mathrm{~h}) 1.32 \mathrm{~h}\end{array}$ & $\begin{array}{l}\text { II } \\
\text { II } \\
\text { II }\end{array}$ \\
\hline $\begin{array}{l}60.86 \\
60.49 \\
57.88 \\
56.18 \\
54.79\end{array}$ & $\begin{array}{r}2 \\
2 \\
2 \\
\frac{2}{20}\end{array}$ & $\begin{array}{l}? \\
2 \\
1 \\
1 \\
?\end{array}$ & $\begin{array}{l}2 \text { III A } \\
2 \text { III A } \\
2 \text { III A } \\
25 \text { III }\end{array}$ & $\begin{array}{l}924.84 \\
926.78 \\
940.51 \\
949.46 \\
956.79\end{array}$ & 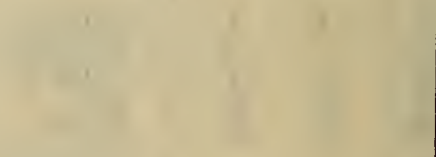 & $\begin{array}{l}\text { I } \\
\text { I } \\
\text { I }\end{array}$ \\
\hline $\begin{array}{l}54.40 \\
40.72 \mathrm{c} \\
39.93 \\
37.78 \\
34.96 \mathrm{c}\end{array}$ & $\begin{array}{r}60 \\
10 \\
5 \\
2 \\
50\end{array}$ & $\begin{array}{c}200 \\
6 \\
3 \\
101 \\
100\end{array}$ & $\begin{array}{l}80 \text { IV E } \\
15 \text { III } \\
6 \text { III A } \\
60 \mathrm{~V} \mathrm{E}\end{array}$ & $\begin{array}{r}22,958.84 \\
23,031.20 \\
035.39 \\
046.81 \\
061.80 \\
068.19\end{array}$ & $\begin{array}{l}(0.00) 1.08 \\
(0.00) 0.90 \\
(0.00) 1.08 \mathrm{~h} \\
(0.00 .0 .29,0.57) 0.69,0.97,1.26 \text {, } \\
\left.\begin{array}{l}1.54 \\
(0.17)\end{array}\right) .94\end{array}$ & $\begin{array}{l}\text { II } \\
\text { II } \\
\text { II } \\
\text { II }\end{array}$ \\
\hline $33.76 \mathrm{c}$ & 300 & 500 & 600 III E & 068.19 & $(0.17) 0.94$ & II \\
\hline $\begin{array}{l}26.19 \\
22.51 \mathrm{c} \\
15.90 \mathrm{c}\end{array}$ & $\begin{array}{r}2 \\
60 \\
10\end{array}$ & $\begin{array}{r}2 \\
100 \\
30\end{array}$ & $\begin{array}{l}130 \text { III E } \\
12 \mathrm{VE}\end{array}$ & $\begin{array}{l}108.55 \\
128.22 \\
163.65\end{array}$ & $\begin{array}{l}(0.00) 1.10 \\
(0.00,0.50,1.00) 0.86,1.36,1.86 \text {, } \\
2.36\end{array}$ & ${ }_{\text {II }}^{\text {II }}$ \\
\hline $\begin{array}{l}11.73 \mathrm{c} \\
4,306.00\end{array}$ & $\begin{array}{l}5 \\
6\end{array}$ & $\begin{array}{l}4 \\
5\end{array}$ & - $\begin{array}{l}4 \text { III A } \\
6 \text { II A }\end{array}$ & $\begin{array}{r}186.05 \\
23,216.90\end{array}$ & $\begin{array}{l}(0.59) 0.96 \mathrm{R} \\
(0.14) 1.49\end{array}$ & \\
\hline
\end{tabular}


TABLE 1.-Wave lengths and Zeeman effects in lanthanum spectra-Continued

\begin{tabular}{|c|c|c|c|c|c|}
\hline $\begin{array}{l}\lambda \text { (air) } \\
\text { I. A. }\end{array}$ & $\begin{array}{l}\text { Intensity } \\
\text { arc spark } \\
\text { B. S. }\end{array}$ & $\begin{array}{l}\text { Arc intensity } \\
\text { and tempera- } \\
\text { ture class } \\
\mathrm{K} \text { and } \mathrm{C}\end{array}$ & $\nu\left(\mathrm{vac}^{-1}\right)$ & Zeeman effect & $\begin{array}{l}\text { Spec- } \\
\text { trum }\end{array}$ \\
\hline $\begin{array}{c}4,304.11 \\
00.62 \\
4,300.44 \\
4,296.05 \mathrm{c} \\
91.00\end{array}$ & $\begin{array}{cc}\text { H. N. R. } & 10 \mathrm{hl} \\
40 & 60 \\
100 & 300 \\
2 & 2\end{array}$ & $\begin{array}{l}3 \\
30 \mathrm{IV} \mathrm{E} \\
200 \mathrm{IV} \mathrm{E}\end{array}$ & $\begin{array}{r}23,227.10 \\
245.94 \\
246.92 \\
270.67 \\
298.06\end{array}$ & $\begin{array}{l}(0.00 \mathrm{~h}) 1.24 \mathrm{~h} \\
(0.73,1.48) 0.00,0.72,1.45,2.18 \\
(0.00) 1.48\end{array}$ & $\mathrm{II}_{\text {II }}^{\text {II }}$ \\
\hline $\begin{array}{l}89.65 \\
89.01 \\
86.97 \mathrm{c} \\
80.27 \\
75.64\end{array}$ & $\begin{array}{ll}\text { H. N. } & \text { R. } \\
\text { H. N. } & \text { R. } \\
80 & 300 \\
60 & 40 \\
50 & 100\end{array}$ & $\begin{array}{l}1 \\
2 \\
150 \mathrm{~V} \mathrm{E} \\
100 \mathrm{I} \\
60 \mathrm{IV} \mathrm{E}\end{array}$ & $\begin{array}{l}305.39 \\
308.87 \\
319.96 \\
356.46 \\
381.75\end{array}$ & 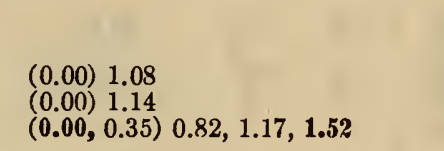 & $\begin{array}{l}\text { I } \\
\text { I } \\
\text { II } \\
\text { II }\end{array}$ \\
\hline $\begin{array}{l}71.14 \\
69.50 \mathrm{c} \\
67.74 \\
63.59 \mathrm{c} \\
62.35\end{array}$ & $\begin{array}{rr}4 & 3 \\
80 & 300 \\
2 & 2 \\
60 & 200 \\
10 & 5\end{array}$ & $\begin{aligned} 150 \mathrm{~V} \mathrm{E} \\
100 \mathrm{VE} \\
15 \mathrm{II} \mathrm{A}\end{aligned}$ & $\begin{array}{l}406.39 \\
415.38 \\
425.04 \\
447.83 \\
454.66\end{array}$ & $\begin{array}{l}(0.00) 1.20 \\
(0.00) 1.12 \\
(0.00 \mathrm{w}) 1.19\end{array}$ & $\begin{array}{l}\text { I } \\
\text { II } \\
\text { III }\end{array}$ \\
\hline $\begin{array}{l}59.51 \\
56.92 \mathrm{c} \\
56.50 \\
52.93 \\
49.99 \mathrm{c}\end{array}$ & $\begin{array}{rr}- & 2 \mathrm{~h} \\
6 & 5 \\
-3 & 3 \\
20 & 4 \\
20 & 100\end{array}$ & $25 \mathrm{VE}$ & $\begin{array}{l}470.30 \\
484.58 \\
486.89 \\
506.61 \\
522.87\end{array}$ & $\begin{array}{l}(0.00) 1.29 \mathrm{R} \\
(0.74) 0.50,0.76,1.02,1.27,1.53 \text {, } \\
1.76\end{array}$ & I II \\
\hline $\begin{array}{l}48.32 \\
41.20 \\
38.59 \\
38.38 \mathrm{c}\end{array}$ & $\begin{array}{rc}- & 2 \\
1 & 15 \mathrm{hl} \\
4 & (?) \\
200 & 400\end{array}$ & $\begin{array}{r}10 \text { III A } \\
400 \text { III E }\end{array}$ & $\begin{array}{l}532.11 \\
571.62 \\
586.13 \\
587.30\end{array}$ & (0.74) $0.56,0.82,1.08,1.34,1.60$ & III \\
\hline $30.95 \mathrm{c}$ & 150 & $30 \mathrm{VE}$ & 628.72 & $\begin{array}{l}1.86 \\
(0.80) 0.65,0.85,1.06,1.26,1.47 \text {, } \\
1.67 \mathrm{ur}\end{array}$ & II \\
\hline $\begin{array}{l}17.56 \mathrm{c} \\
16.54 \\
10.22 \\
07.61 \\
04.03\end{array}$ & $\begin{array}{rc}40 & 200 \\
4 & 3 \\
4 & 50 \mathrm{hl} \\
2 & 101 \\
40 & 100\end{array}$ & $100 \mathrm{VE}$ & $\begin{array}{l}703.74 \\
709.47 \\
745.06 \\
759.79 \\
780.02\end{array}$ & $\begin{array}{l}(0.00) 1.22 \\
(0.00 \mathrm{~h}) 1.02 \mathrm{~h} \\
(0.27) 0.68 \mathrm{~h} \\
(0.00) 1.48\end{array}$ & $\begin{array}{l}\text { II } \\
\text { II } \\
\text { II } \\
\text { II }\end{array}$ \\
\hline $\begin{array}{c}4,201.50 \\
4,196.55 \mathrm{c} \\
94.36 \\
93.34 \\
92.72\end{array}$ & \begin{tabular}{|cc} 
& $6 \mathrm{~h}$ \\
150 & 250 \\
4 & $30 \mathrm{~h}$ \\
1 & 5 \\
H. N. R. &
\end{tabular} & $\begin{array}{l}300 \text { III } \mathrm{E} \\
2\end{array}$ & $\begin{array}{l}794.34 \\
822.41 \\
834.85 \\
840.64 \\
844.17\end{array}$ & $\begin{array}{l}(0.32,0.64) 0.51,0.82,1.13,1.44 \\
(0.00 \mathrm{~h})(1.06 \mathrm{~h}) \\
(0.25) 0.64\end{array}$ & $\begin{array}{l}\text { II } \\
\text { II } \\
\text { II } \\
\text { II }\end{array}$ \\
\hline $\begin{array}{l}92.35 \mathrm{c} \\
87.31 \\
80.97 \\
77.48 \\
72.32\end{array}$ & $\begin{array}{rc}40 & 100 \\
50 & 30 \\
2 & 121 \\
15 & 10 \\
6 & 4\end{array}$ & $\begin{array}{l}80 \text { V E } \\
125 \text { I } \\
30 \text { I } \\
10 \text { III A }\end{array}$ & $\begin{array}{l}846.27 \\
874.98 \\
911.18 \\
931.15 \\
960.75\end{array}$ & $\begin{array}{l}(0.00) 1.06 \\
(0.00 \mathrm{w}) 1.16 \mathrm{~A}^{2} \\
(0.00) 1.50\end{array}$ & $\begin{array}{l}\text { II } \\
\text { II } \\
\text { II } \\
\text { I }\end{array}$ \\
\hline $\begin{array}{l}71.13 \\
63.31 \\
61.94 \\
60.26 \\
57.52\end{array}$ & $\begin{array}{rc}5 & 4 \\
5 & 4 \\
2 & 8 \mathrm{~h} \\
20 & 10 \\
6 & 5\end{array}$ & $\begin{array}{l}8 \text { III A } \\
8 \text { III A } \\
30 \text { I } \\
10 \text { II A }\end{array}$ & $\begin{array}{r}23,967.58 \\
24,012.60 \\
020.51 \\
030.21 \\
046.04\end{array}$ & $\begin{array}{l}(0.00 \mathrm{~h}) 1.02 \\
(0.00 \mathrm{~W}) 1.65 \mathrm{w}\end{array}$ & $\begin{array}{l}\text { I } \\
\text { I } \\
\text { II } \\
\text { I }\end{array}$ \\
\hline $\begin{array}{l}54.59 \\
52.78 \\
51.98 \mathrm{c} \\
50.24 \\
48.2\end{array}$ & $\begin{array}{rc}- & 2 \mathrm{~h} \\
40 & 100 \\
100 & 250 \\
2 & 2 \\
- & 4 \mathrm{~h}\end{array}$ & $\begin{array}{l}40 \mathrm{IV}(?) \mathrm{E} \\
300 \mathrm{III} \mathrm{E}\end{array}$ & $\begin{array}{l}053.00 \\
073.49 \\
078.13 \\
088.22 \\
100.1\end{array}$ & $\begin{array}{l}(0.43,0.84) 0.27,0.67,1.08,1.48 \\
(0.29) 0.50,0.77\end{array}$ & $\begin{array}{r}\text { II } \\
\text { II } \\
\text { II } \\
\text { II }\end{array}$ \\
\hline $\begin{array}{l}44.36 \\
43.92 \\
43.77 \\
41.73 \mathrm{c} \\
37.91\end{array}$ & $\begin{array}{rr}4 & 2 \\
5 & (?) \\
6 & 15 \\
80 & 200 \\
- & 2\end{array}$ & $\begin{array}{c}12 \text { III A } \\
\left\{\begin{array}{l}40 \\
50\end{array}\right\} \text { IV E }\end{array}$ & $\begin{array}{l}122.40 \\
124.96 \\
125.83 \\
137.72 \\
160.00\end{array}$ & $(0.00 \mathrm{~W}) 1.50 \mathrm{~W}$ & $\begin{array}{l}\text { I } \\
\text { I } \\
\text { II } \\
\text { II } \\
\text { II }\end{array}$ \\
\hline $\begin{array}{r}37.05 \\
33.33 \\
32.50 \\
31.74 \\
4,123.23\end{array}$ & \begin{tabular}{cc}
20 & 10 \\
\hdashline & $\begin{array}{c}6 \mathrm{hl} \\
1\end{array}$ \\
$\begin{array}{c}10 \mathrm{hl} \\
5 \mathrm{~h}\end{array}$ \\
200 & 400
\end{tabular} & 600 III $\mathrm{E}$ & $\begin{array}{r}165.02 \\
186.77 \\
191.63 \\
196.08 \\
24,246.01\end{array}$ & $\begin{array}{l}(0.00) 1.22 \\
(0.00) 0.97 \\
(0.00 \mathrm{~h}) 0.83 \mathrm{~h} \\
(0.00 \mathrm{~h}) 1.13 \mathrm{~h} \\
(0.00) 1.05\end{array}$ & $\begin{array}{l}\text { I } \\
\text { II } \\
\text { II } \\
\text { II } \\
\text { II }\end{array}$ \\
\hline
\end{tabular}


TABLE 1.-Wave lengths and Zeeman effects in lanthanum spectra-Continued

\begin{tabular}{|c|c|c|c|c|c|c|}
\hline $\begin{array}{l}\lambda \text { (air) } \\
\text { I. A. }\end{array}$ & $\begin{array}{c}\text { Int } \\
\text { arc } \\
I\end{array}$ & $\begin{array}{l}\text { nsity } \\
\text { spark } \\
\text { S. }\end{array}$ & $\begin{array}{l}\text { Arc intensity } \\
\text { and tempera- } \\
\text { ture class } \\
\mathrm{K} \text { and } \mathrm{C}\end{array}$ & $\begin{array}{c}\nu \text { (vac.) } \\
\mathrm{cm}^{-1}\end{array}$ & Zeeman effect & $\begin{array}{l}\text { Spec- } \\
\text { trum }\end{array}$ \\
\hline $\begin{array}{r}4,117.67 \\
15.35 \\
13.28 \\
09.80 \\
09.48\end{array}$ & $\begin{array}{r}\frac{8}{2} \\
10 \\
6\end{array}$ & $\begin{array}{c}5 \\
1 \mathrm{~h} \\
40 \mathrm{l} \\
6 \\
3\end{array}$ & $\begin{array}{l}20 \text { III } \\
20 \text { I A } \\
15 \text { II A }\end{array}$ & $\begin{array}{r}24,278.75 \\
292.44 \\
304.66 \\
325.24 \\
327.14\end{array}$ & $\begin{array}{l}(0.00) 0.93 \\
(0.00 \mathrm{~h}) 1.09 \mathrm{~h} \\
(0.00) 1.04 \\
(0.00) 1.26\end{array}$ & ${ }_{\text {I II }}^{\text {I }}$ \\
\hline $\begin{array}{c}04.87 \\
4,101.01 \\
4,099.54 \mathrm{c} \\
98.73 \\
90.40\end{array}$ & $\begin{array}{r}\frac{30}{40} \\
1 \\
2\end{array}$ & $\begin{array}{c}20 \\
3 \mathrm{~h} \\
150 \\
5 \\
1\end{array}$ & $\begin{array}{l}60 \mathrm{I} \\
60 \mathrm{~V} \mathrm{E}\end{array}$ & $\begin{array}{l}354.46 \\
377.38 \\
386.12 \\
390.94 \\
440.61\end{array}$ & $\begin{array}{l}(0.08,0.25) 0.53,0.69,0.85 \mathrm{ur} \\
(0.00) 0.77\end{array}$ & $\begin{array}{l}\text { I II } \\
\text { II } \\
\text { II }\end{array}$ \\
\hline $\begin{array}{l}89.61 \\
86.72 \\
79.17 \\
77.35 \mathrm{c} \\
76.71\end{array}$ & $\begin{array}{r}25 \\
200 \\
20 \\
200 \\
10\end{array}$ & $\begin{array}{r}12 \\
300 \\
10 \\
300 \\
40\end{array}$ & $\begin{aligned} & 50 \text { I } \\
& 400 \text { III E } \\
& 40 \text { I } \\
& 400 \text { III E } \\
& 30 \text { IV E }\end{aligned}$ & $\begin{array}{l}445.33 \\
462.62 \\
507.90 \\
518.84 \\
522.68\end{array}$ & $\begin{array}{l}(0.00) 0.93 \\
(0.32) 0.79 \mathrm{w} \\
(\mathbf{0 . 0 0 , 0 . 3 2 )} 0.57,0.89, \mathbf{1 . 2 0} \\
(\mathbf{0 . 0 0}, 0.31,0.62) 0.79,1.08,1.38 \\
\mathbf{1 . 6 9}\end{array}$ & I II \\
\hline $\begin{array}{l}67.39 \mathrm{c} \\
65.58 \\
64.79 \\
60.33 \\
.58 .08\end{array}$ & $\begin{array}{r}60 \\
15 \\
25 \\
30 \\
2\end{array}$ & $\begin{array}{r}100 \\
6 \\
15 \\
20 \\
51\end{array}$ & $\begin{array}{l}125 \text { IV } \mathrm{E} \\
30 \mathrm{II} \\
50 \mathrm{II} \\
60 \mathrm{II}\end{array}$ & $\begin{array}{l}578.88 \\
589.82 \\
594.60 \\
621.61 \\
635.26\end{array}$ & $\begin{array}{l}(0.00 \mathrm{~W}) 1.14 \mathrm{~A}^{2} \\
(0.00 \mathrm{~W}) 1.03 \mathrm{~A}^{1} \\
(0.00 \mathrm{~W}) 1.10 \mathrm{~A}^{1} \\
(0.45) 1.06,1.47 \mathrm{us}\end{array}$ & $\begin{array}{l}\text { II } \\
\text { I }_{\text {II }}^{\text {II }}\end{array}$ \\
\hline $\begin{array}{l}50.08 \mathrm{c} \\
42.91 \\
40.97 \\
37.21 \\
36.59 \mathrm{c}\end{array}$ & $\begin{array}{r}50 \\
150 \\
2 \\
25 \\
7\end{array}$ & $\begin{array}{c}200 \\
300 \\
1 \\
10 \\
15 \mathrm{~d}\end{array}$ & $\begin{array}{l}60 \mathrm{~V} \mathrm{E} \\
300 \mathrm{IV} \mathrm{E} \\
50 \mathrm{I} \\
8 \mathrm{~V} \mathrm{E}\end{array}$ & $\begin{array}{l}683.92 \\
727.70 \\
739.57 \\
762.61 \\
766.41\end{array}$ & 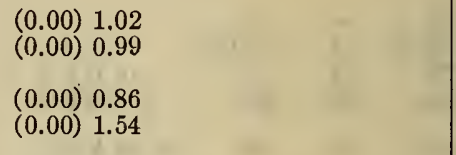 & $\begin{array}{l}\text { II } \\
\text { II } \\
\text { III }_{\text {II }}\end{array}$ \\
\hline $\begin{array}{l}31.68 \\
25.87 \\
23.58 \mathrm{c} \\
20.19 \\
15.39\end{array}$ & $\begin{array}{r}100 \\
20 \\
10 \\
25\end{array}$ & $\begin{array}{l}300 \\
50 \\
40 \\
2 \mathrm{~h} \\
15\end{array}$ & $\begin{array}{l}300 \text { III E } \\
40 \text { IV E } \\
15 \text { IV E } \\
50 \mathrm{I}\end{array}$ & $\begin{array}{l}796.57 \\
832.36 \\
846.49 \\
867.44 \\
897.17\end{array}$ & $\begin{array}{l}(0.00) 1.15 \\
(0.00 \mathrm{~W}) 1.37 \mathrm{~A}^{2} \\
(0.00) 1.07 \\
(0.10,0.31) 0.90,1.10,1.30,1.48 \mathrm{ur}\end{array}$ & $\begin{array}{l}\text { II } \\
\text { II } \\
\text { II } \\
\text { II }\end{array}$ \\
\hline $\begin{array}{r}07.64 \\
4,001.38 \\
3,995.74 \mathrm{c} \\
94.50 \mathrm{c} \\
88.51 \mathrm{c}\end{array}$ & $\begin{array}{r}1 \\
2 \\
200 \\
4 \\
300\end{array}$ & $\begin{array}{c}7 \mathrm{~h} \\
2 \\
400 \\
10 \\
500\end{array}$ & $\begin{array}{l}600 \text { III E } \\
800 \text { III E }\end{array}$ & $\begin{array}{r}945.32 \\
24,984.34 \\
25,019.61 \\
027.37 \\
064.96\end{array}$ & $\begin{array}{l}(0.00 \mathrm{~W}) 1.01 \mathrm{~h} \\
(0.30) 0.90 \mathrm{~W} \\
(0.57 \mathrm{~h}) 1.25 \mathrm{~h} \\
(0.00) 1.32\end{array}$ & $\begin{array}{l}\text { II } \\
\text { II } \\
\text { II } \\
\text { II }\end{array}$ \\
\hline $\begin{array}{l}81.36 \\
79.08 \\
63.04 \\
62.03 \\
58.53\end{array}$ & $\begin{array}{l}2 \\
1 \\
1 \\
1 \\
-\end{array}$ & $\begin{array}{r}101 \\
81 \\
51 \\
101 \\
2\end{array}$ & I. & $\begin{array}{l}109.97 \\
124.35 \\
226.04 \\
232.47 \\
254.78\end{array}$ & $\begin{array}{l}(0.38) 0.98 \mathrm{~h} \text { us } \\
(0.00) 1.26 \\
(0.00 \mathrm{w}) 0.82 \mathrm{~h} \\
(0.00) 1.10 \mathrm{~h}\end{array}$ & $\begin{array}{l}\text { II } \\
\text { II } \\
\text { II } \\
\text { II } \\
\text { II }\end{array}$ \\
\hline $\begin{array}{l}57.25 \\
56.07 \\
55.21 \\
53.67 \\
53.36\end{array}$ & $\frac{-1}{10}$ & $\begin{array}{l}2 \\
4 \\
3 \mathrm{~h} \\
5 \\
2\end{array}$ & $40 \mathrm{II}$ & $\begin{array}{l}262.95 \\
270.48 \\
275.98 \\
285.83 \\
287.81\end{array}$ & $\begin{array}{l}(0.00 \mathrm{~h}) 1.29 \mathrm{~h} \\
(0.00) 1.15 \\
(0.00) 1.06\end{array}$ & $\begin{array}{l}\text { II } \\
\text { II } \\
\text { II } \\
\text { II }\end{array}$ \\
\hline $\begin{array}{l}51.43 \\
49.10 \mathrm{c} \\
44.15 \\
39.85 \\
36.22\end{array}$ & $\begin{array}{r}1 \\
400 \\
2 \\
2 \\
20\end{array}$ & $\begin{array}{c}3 \mathrm{~h} \\
600 \\
3 \\
201 \\
50\end{array}$ & $\begin{array}{l}1000 \text { III E } \\
60 \text { IV E }\end{array}$ & $\begin{array}{l}300.16 \\
315.09 \\
346.86 \\
374.52 \\
397.92\end{array}$ & $\begin{array}{l}(0.00) 1.13 \mathrm{w} \\
(0.00) 1.18 \mathrm{~h} \\
(0.21) 1.27 \mathrm{~h} \\
(0.00,0.25,0.48) 0.86,1.10,1.34,1.58\end{array}$ & $\begin{array}{l}\text { II } \\
\text { II } \\
\text { II } \\
\text { II } \\
\text { II }\end{array}$ \\
\hline $\begin{array}{l}: 32.53 \\
30.47 \\
29.22 \mathrm{c} \\
27.56 \\
25.09\end{array}$ & $\begin{array}{r}\frac{3}{100} \\
30 \\
1\end{array}$ & $\begin{array}{r}101 \\
3 \\
300 \\
10 \\
5\end{array}$ & $\begin{array}{l}500 \mathrm{III} \\
80 \mathrm{I}\end{array}$ & $\begin{array}{l}421.75 \\
435.07 \\
443.17 \\
453.92 \\
469.94\end{array}$ & $\begin{array}{l}(0.44) 1.06 \mathrm{~B} \\
(0.00) 0.92 \\
(0.00) 1.20 \\
(0.00) 0.76\end{array}$ & II \\
\hline $\begin{array}{l}24.69 \\
21.54 \mathrm{c} \\
16.05 \mathrm{c} \\
10.81 \\
3,902.57\end{array}$ & $\begin{array}{r}70 \\
80 \\
4 \\
5\end{array}$ & $\begin{array}{l}3 \\
200 \\
300 \\
101 \\
3\end{array}$ & $\begin{array}{l}300 \text { III E } \\
400 \text { III E } \\
15 \text { IV (?) E } \\
20 \text { II }\end{array}$ & $\begin{array}{l}472.53 \\
492.99 \\
528.73 \\
562.94 \\
616.91\end{array}$ & $\begin{array}{l}(0.00 \mathrm{~h}) 0.72 \mathrm{~h} \\
(\boldsymbol{\theta . 0 0}, 0.66) 0.52,1.18,1.84 \\
(0.38) 0.51,0.88 \\
(0.00) 1.04\end{array}$ & $\begin{array}{l}\text { II } \\
\text { II } \\
\text { II }\end{array}$ \\
\hline $\begin{array}{r}3,898.60 \\
97.43 \\
95.65 \\
92.47 \\
92.05 \\
3,886.37\end{array}$ & $\begin{array}{r}8 \\
2 \\
- \\
60\end{array}$ & $\begin{array}{l}3 \\
3 \\
150\end{array}$ & $\begin{array}{l}40 \mathrm{II} \\
8 \mathrm{IV} \\
400 \mathrm{III} \mathrm{E}\end{array}$ & $\begin{array}{r}642.99 \\
650.70 \\
662.42 \\
683.37 \\
686.15 \\
25,723.69\end{array}$ & $\begin{array}{l}(0.00 \mathrm{~h}) 1.4 \mathrm{~h} \\
(0.00 \mathrm{~W}) 1.62 \mathrm{~A}^{2}\end{array}$ & $\begin{array}{l}\text { I } \\
\text { II } \\
\text { Fe(?) } \\
\text { II } \\
\text { II }\end{array}$ \\
\hline
\end{tabular}


TABLE 1.-Wave lengths and Zeeman effects in lanthanum spectra-Continued

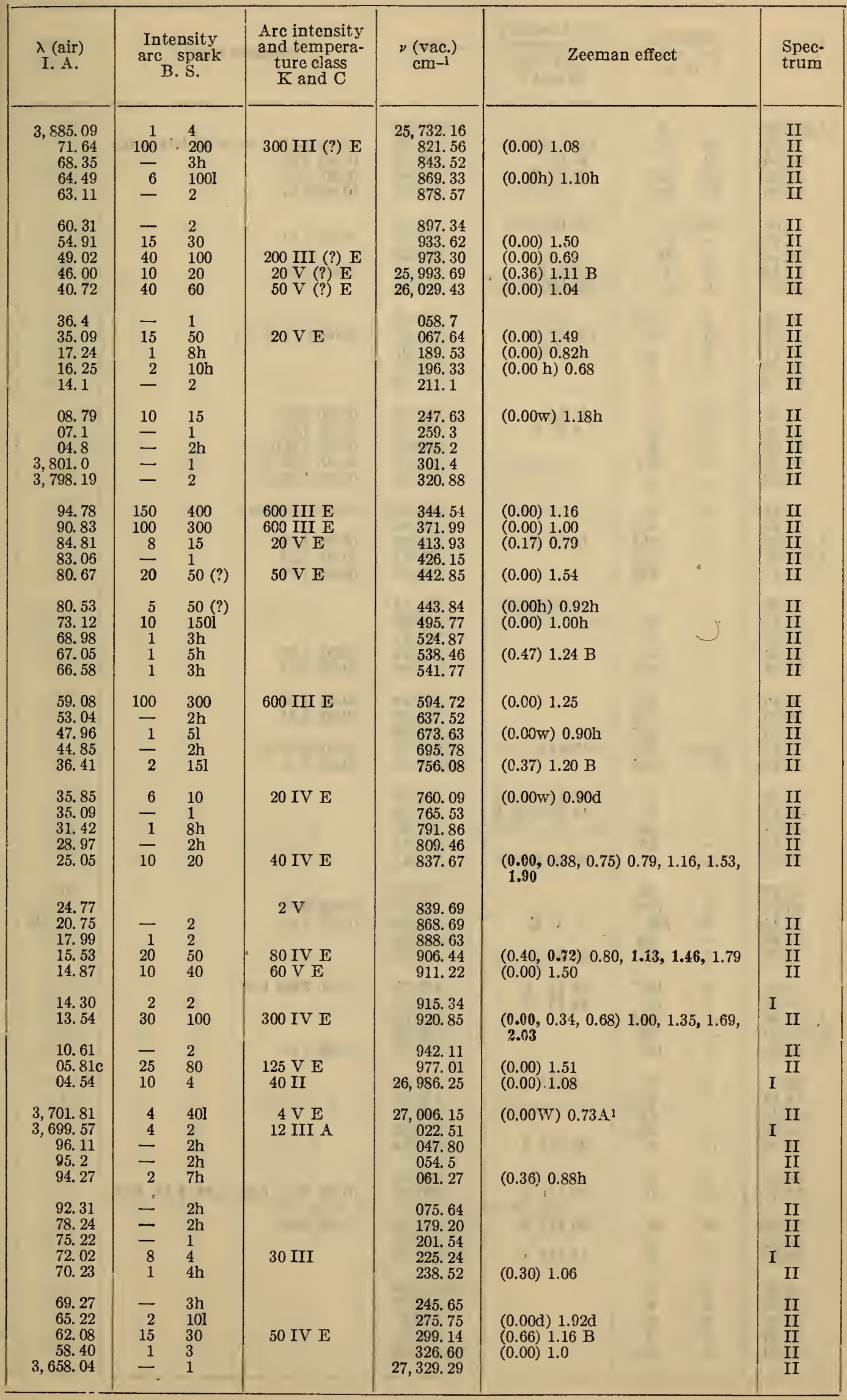


TABLE 1.-Wave lengths and Zeeman effects in lanthanum spectra-Continued

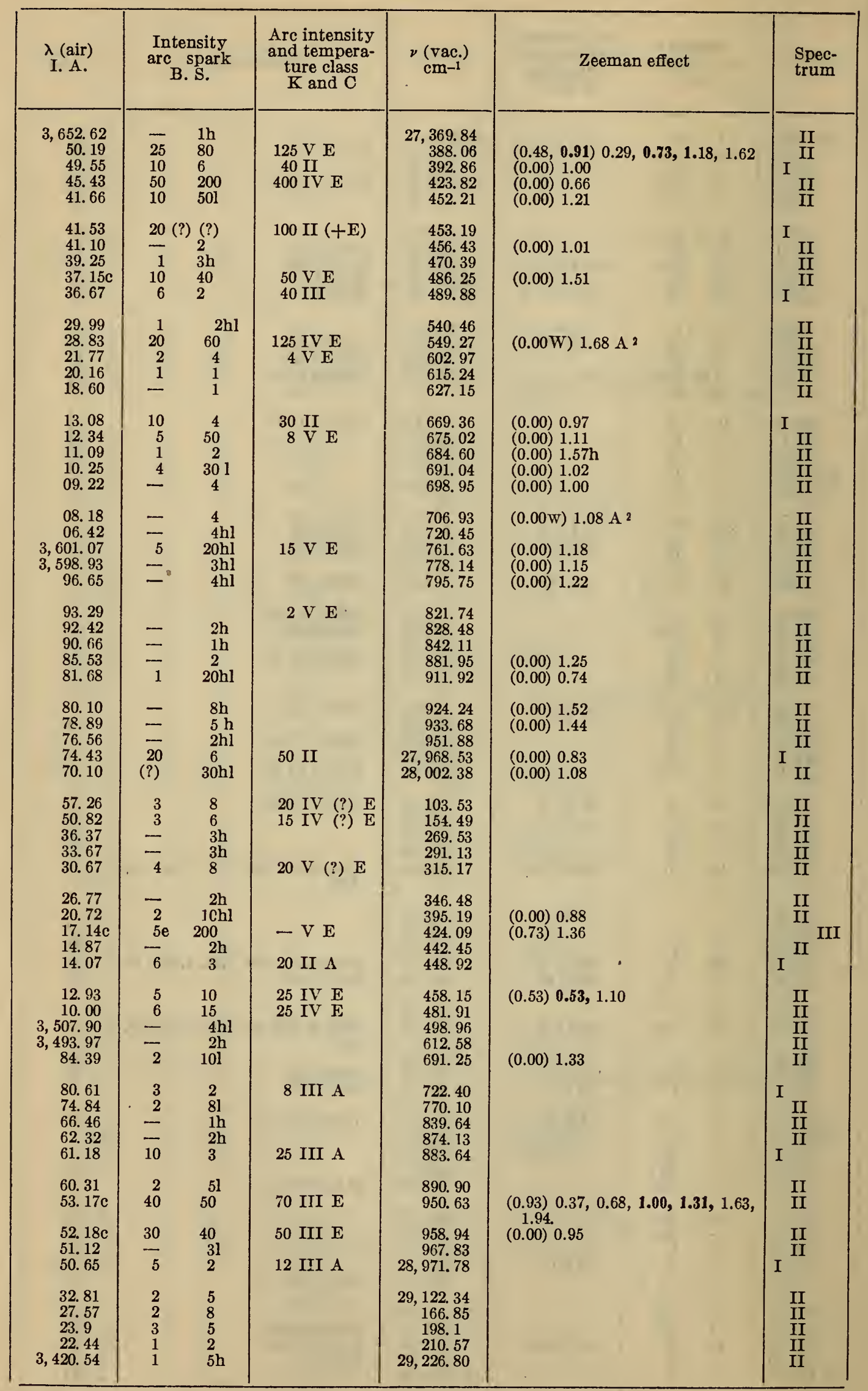


TABLE 1.-Wave lengths and Zeeman effects in lanthanum spectra-Continued

\begin{tabular}{|c|c|c|c|c|c|c|}
\hline $\begin{array}{l}\lambda \text { (air) } \\
\text { I. A. }\end{array}$ & \multicolumn{2}{|c|}{$\begin{array}{l}\text { Intensity } \\
\text { arc spark } \\
\text { B. S. }\end{array}$} & $\begin{array}{l}\text { Arc intensity } \\
\text { and tempera- } \\
\text { ture class } \\
\mathrm{K} \text { and } \mathrm{C}\end{array}$ & $\begin{array}{l}\nu\left(\mathrm{vac}_{-}\right) \\
\mathrm{cm}^{-1}\end{array}$ & Zeeman effect & $\begin{array}{l}\text { Spec- } \\
\text { trum }\end{array}$ \\
\hline $\begin{array}{r}3,411.76 \\
07.00 \\
3,404.53 \\
3,398.29 \\
97.77\end{array}$ & $\begin{array}{r}2 \\
1 \\
10 \\
1 \\
4\end{array}$ & $\begin{array}{c}20 \mathrm{hl} \\
8 \mathrm{hl} \\
3 \\
2 \mathrm{~h} \\
40 \mathrm{hl}\end{array}$ & $\begin{array}{c}15 \text { III A } \\
4 \mathrm{~V} \mathrm{E}\end{array}$ & $\begin{array}{r}29,302.01 \\
342.95 \\
364.24 \\
418.15 \\
422.65\end{array}$ & $\begin{array}{l}(0.00 \mathrm{w}) 1.48 \\
(0.00) 1.50 \\
(0.24) 0.85\end{array}$ & II ${ }_{\text {II }}^{\text {II }}$ \\
\hline $\begin{array}{l}92.94 \\
90.40 \\
88.61 \\
81.42 \\
80.91\end{array}$ & $\begin{array}{l}1 \\
1 \\
6\end{array}$ & $\begin{array}{l}4 \mathrm{~h} \\
4 \mathrm{~h} \\
2 \\
300\end{array}$ & $\begin{array}{r}12 \text { II A } \\
15 \text { II A } \\
400 \text { III E }\end{array}$ & $\begin{array}{l}464.54 \\
486.61 \\
502.18 \\
564.92 \\
569.36\end{array}$ & $(0.00,0.37) 0.75,1.11,1.47$ & $\stackrel{\text { II }}{\text { II }}_{\text {II }}^{\text {II }}$ \\
\hline $\begin{array}{l}76.33 \\
74.89 \\
68.36 \\
64.88 \\
62.04\end{array}$ & $\begin{array}{r}40 \\
1 \\
3 \\
2 \\
7\end{array}$ & $\begin{array}{r}50 \\
\frac{3}{3} \\
\frac{3}{3}\end{array}$ & 12 III A & $\begin{array}{l}609.49 \\
622.12 \\
679.54 \\
710.24 \\
735.33\end{array}$ & $(0.00) 0.88$ & $\begin{array}{l}\text { II } \\
\text { II } \\
\text { I } \\
\text { I }\end{array}$ \\
\hline $\begin{array}{l}57.50 \\
51.89 \\
49.82 \\
44.56 \\
42.23\end{array}$ & $\begin{array}{r}\frac{5}{2} \\
150 \\
10\end{array}$ & $\begin{array}{r}2 \\
\frac{3}{200} \\
5\end{array}$ & $\begin{array}{l}7 \text { III A } \\
3 \text { III A } \\
300 \text { III E } \\
20 \text { II A }\end{array}$ & $\begin{array}{l}775.54 \\
825.37 \\
843.80 \\
890.73 \\
911.57\end{array}$ & 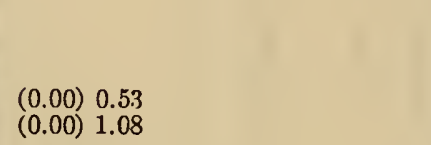 & $\begin{array}{l}\text { I } \\
\text { II } \\
\text { III } \\
\text { I }\end{array}$ \\
\hline $\begin{array}{l}37.49 \\
29.07 \\
26.21 \\
25.33 \\
10.62\end{array}$ & $\begin{array}{r}200 \\
2 \\
1 \\
1 \\
1\end{array}$ & $\begin{array}{r}300 \\
8 \\
5 \\
3 \\
4\end{array}$ & 500 III E & $\begin{array}{r}29,954.05 \\
30,029.81 \\
055.63 \\
063.59 \\
197.16\end{array}$ & $(0.00 \mathrm{w}) 1.12 \mathrm{Al}^{1}$ & $\begin{array}{l}\text { II } \\
\text { II } \\
\text { II } \\
\text { II } \\
\text { II }\end{array}$ \\
\hline $\begin{array}{c}05.98 \\
3,303.11 \mathrm{c} \\
3,298.72 \\
97.15 \\
94.44\end{array}$ & $\frac{7}{100}$ & $\begin{array}{c}8 \\
150 \\
5 \mathrm{~h} \\
3 \\
10\end{array}$ & $\begin{array}{l}10 \text { IV E } \\
200 \text { III }\end{array}$ & $\begin{array}{l}230.40 \\
265.82 \\
306.09 \\
320.52 \\
345.46\end{array}$ & $\begin{array}{l}(0.00) 1.77 \mathrm{R} \\
(1.00) 0.51,1.51\end{array}$ & $\begin{array}{l}\text { II } \\
\text { II } \\
\text { II } \\
\text { II } \\
\text { II }\end{array}$ \\
\hline $\begin{array}{l}83.95 \\
77.83 \\
67.31 \\
65.67 \mathrm{c} \\
63.98\end{array}$ & $\begin{array}{r}1 \\
1 \\
80 \\
2\end{array}$ & $\begin{array}{c}8 \mathrm{~h} \\
4 \\
3 \\
100 \\
5\end{array}$ & 150 III E & $\begin{array}{l}442.39 \\
499.23 \\
597.43 \\
612.79 \\
628.64\end{array}$ & $(0.48,0.80) 0.82,1.17,1.51,1.86$ & $\begin{array}{l}\text { II } \\
\text { II } \\
\text { II } \\
\text { II } \\
\text { II }\end{array}$ \\
\hline $\begin{array}{l}56.60 \\
53.41 \\
49.35 \\
47.06 \\
45.13\end{array}$ & $\begin{array}{r}2 \\
2 \\
60 \\
5 \\
100\end{array}$ & $\begin{array}{c}1 \\
10 \mathrm{~h} \\
80 \\
2 \\
150\end{array}$ & $\begin{array}{l}100 \mathrm{III} \mathrm{E} \\
8 \mathrm{II} \mathrm{A} \\
200 \mathrm{III} \mathrm{E}\end{array}$ & $\begin{array}{l}698.05 \\
728.15 \\
766.54 \\
788.24 \\
803.55\end{array}$ & $\begin{array}{l}(0.00) 0.65 \\
(0.00,0.50), 0.46,0.94,1.44 \\
(0.00) 1.04\end{array}$ & $\begin{array}{l}\text { I } \\
\text { II } \\
\text { II } \\
\text { II }\end{array}$ \\
\hline $\begin{array}{l}35.66 \\
26.03 \\
24.71 \\
17.12 \\
15.81\end{array}$ & $\begin{array}{r}3 \\
1 \\
2 \\
10\end{array}$ & $\begin{array}{l}2 \\
2 \\
1 \\
8 \mathrm{~h} \\
2\end{array}$ & 5 III A & $\begin{array}{r}896.71 \\
30,988.93 \\
31,001.62 \\
074.76 \\
087.42\end{array}$ & $(0.00) 1.13$ & $\begin{array}{l}\text { II } \\
\text { II } \\
\text { II } \\
\text { I }\end{array}$ \\
\hline $\begin{array}{r}12.56 \\
09.13 \\
08.13 \\
05.75 \\
3,204.55\end{array}$ & $\begin{array}{l}1 \\
2 \\
1 \\
3 \\
1\end{array}$ & $\begin{array}{l}5 \\
6 \\
6 \\
4 \\
3\end{array}$ & 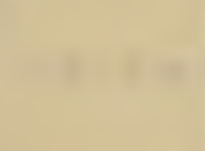 & $\begin{array}{l}118.86 \\
152.12 \\
161.83 \\
184.96 \\
196.64\end{array}$ & t & $\begin{array}{l}\text { II } \\
\text { II } \\
\text { II } \\
\text { II } \\
\text { II }\end{array}$ \\
\hline $3,194.70$ & - & 2 & & 292.83 & & II \\
\hline $93.02 \mathrm{c}$ & $15 \mathrm{~d}$ & 25 & $\begin{array}{l}10(?) \text { IV } \mathrm{E} \\
15(?) \text { IV } \mathrm{E}\end{array}$ & 309.29 & $(0.00,0.96) 0.61,1.58,2.54$ & II \\
\hline $\begin{array}{l}91.39 \\
79.78 \\
75.99\end{array}$ & $\begin{array}{l}1 \\
4 \\
8\end{array}$ & $\begin{array}{c}10 \mathrm{~h} \\
2 \\
2\end{array}$ & $\begin{array}{l}8 \mathrm{III} \mathrm{A}_{15 \mathrm{II} \mathrm{A}} \\
\text {. }\end{array}$ & $\begin{array}{l}325.28 \\
439.65 \\
477.17\end{array}$ & & ${ }_{\mathrm{I}}^{\mathrm{II}}$ \\
\hline $\begin{array}{l}74.88 \\
71.68 \mathrm{c} \\
66.26 \\
65.19 \\
60.56\end{array}$ & $\begin{array}{l}1 \\
8 \mathrm{e} \\
1 \\
1 \\
1\end{array}$ & $\begin{array}{c}10 \mathrm{hl} \\
300 \\
2 \\
4 \\
3\end{array}$ & $-\mathrm{VE}$ & $\begin{array}{l}488.18 \\
519.94 \\
573.90 \\
584.57 \\
630.84\end{array}$ & $(0.37) 0.99,1.76$ & $\begin{array}{l}\text { II } \\
\text { III } \\
\text { II } \\
\text { II }\end{array}$ \\
\hline $\begin{array}{r}57.58 \\
56.35 \\
48.51 \\
45.7 \\
3,142.76\end{array}$ & $\begin{array}{r}\frac{1}{4} \\
1 \\
30\end{array}$ & $\begin{array}{c}2 \\
2 \\
2 \\
2 \mathrm{~h} \\
40\end{array}$ & $\begin{array}{r}4 \mathrm{III} \mathrm{A} \\
50 \mathrm{IV} \mathrm{E}\end{array}$ & $\begin{array}{r}660.69 \\
673.02 \\
751.89 \\
780.3 \\
31,809.98\end{array}$ & ( & $\begin{array}{l}\text { II } \\
\text { II } \\
\text { II } \\
\text { II }\end{array}$ \\
\hline
\end{tabular}


TABLE 1.-Wave lengths and Zeeman effects in lanthanum spectra-Continued

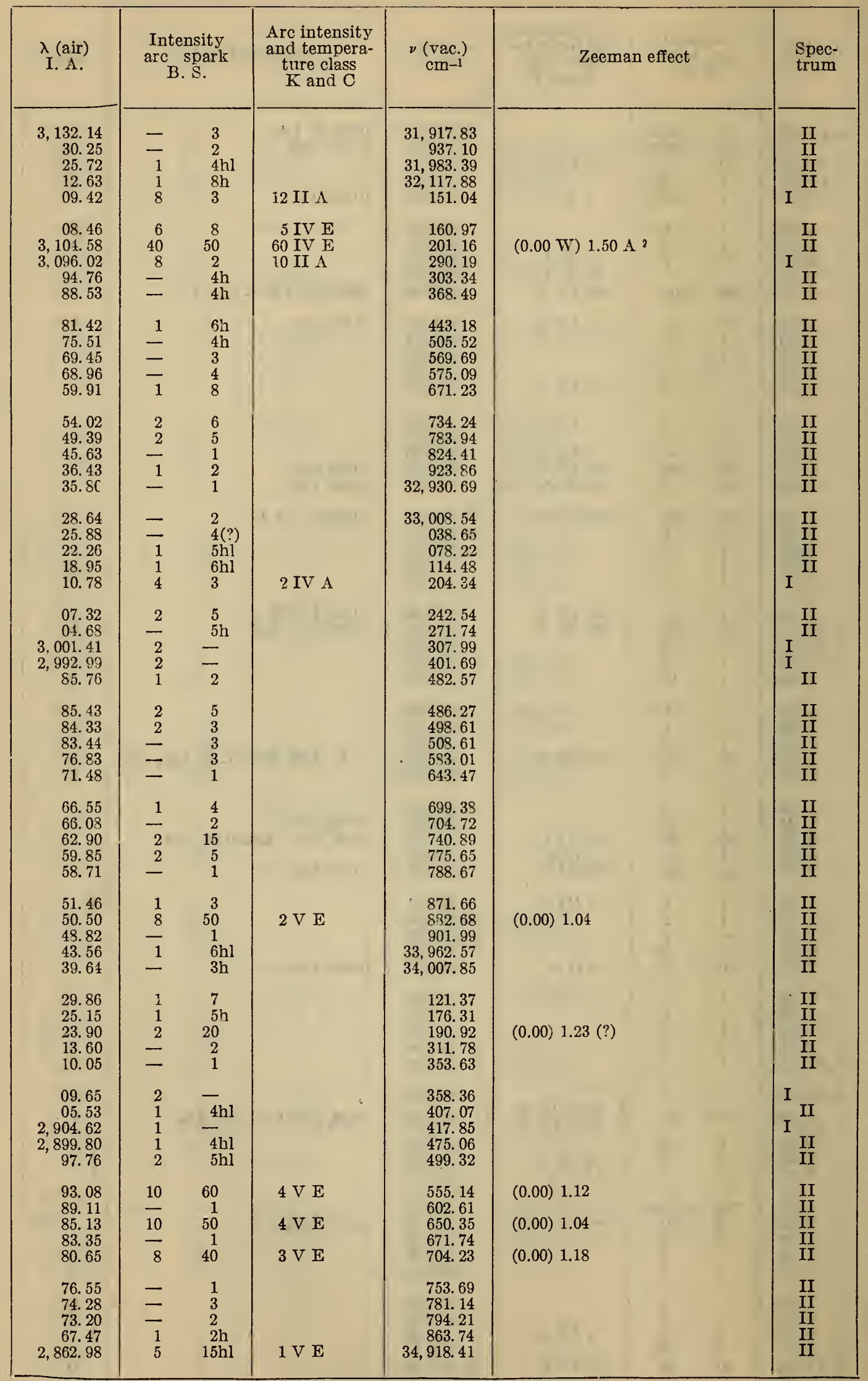


TABLE 1.-Wave lengths and Zeeman effects in lanthanum spectra-Continued

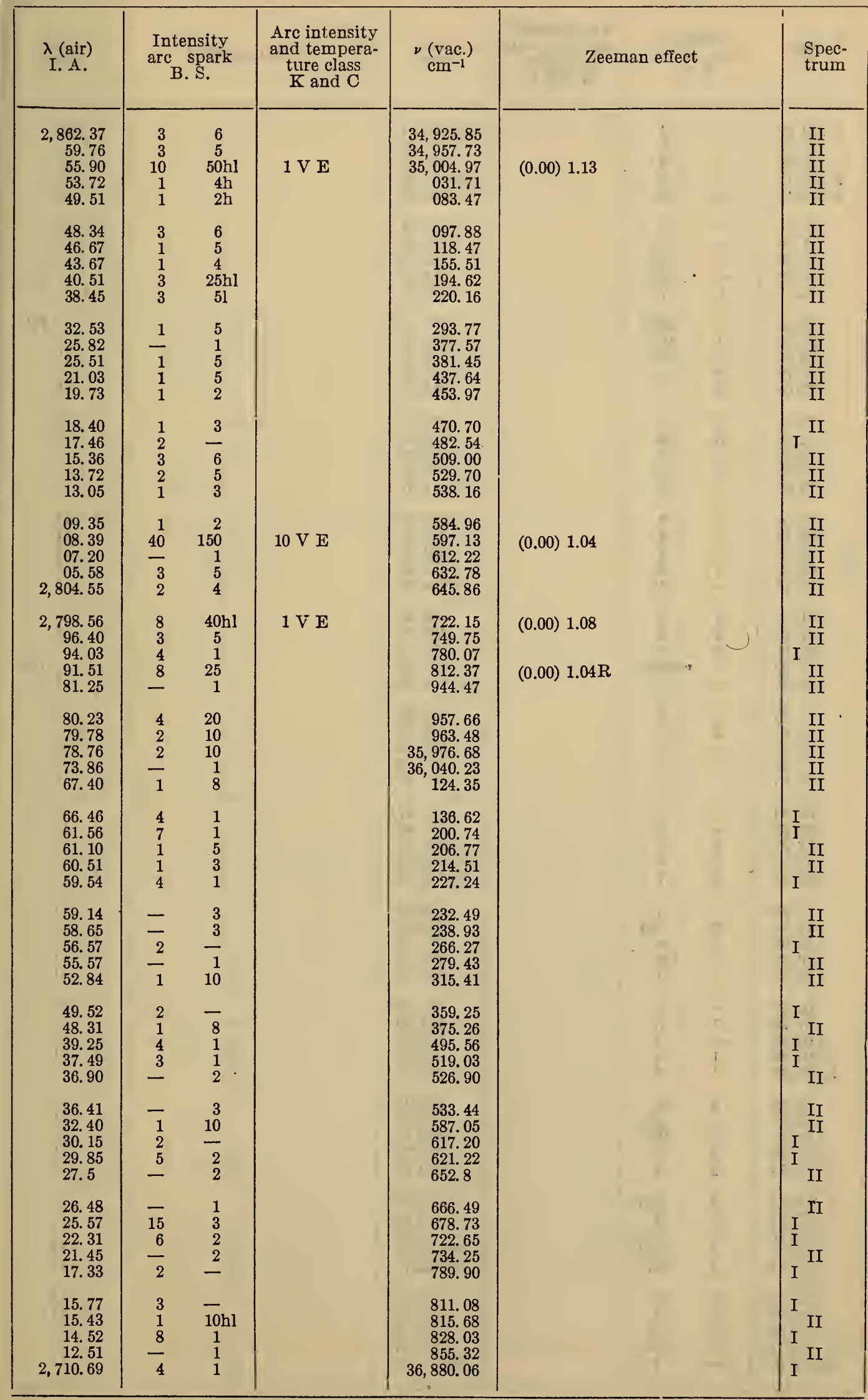


TABLE 1.-Wave lengths and Zeeman effects in lanthanum spectra-Continued

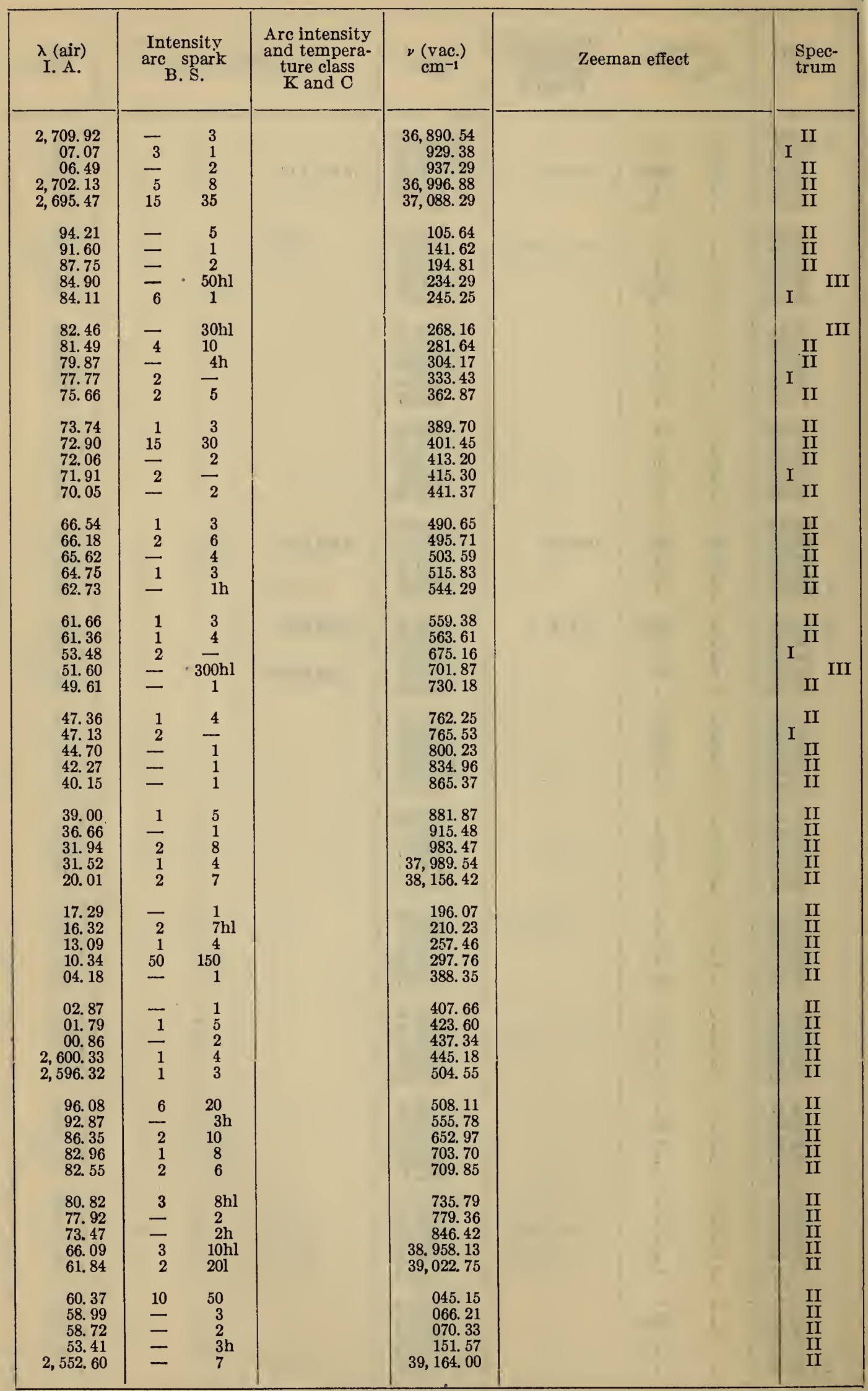


TABLE 1.-Wave lengths and Zeeman effects in lanthanum spectra-Continued

\begin{tabular}{|c|c|c|c|c|c|c|}
\hline $\begin{array}{c}\lambda \text { (air) } \\
\text { I. A. }\end{array}$ & $\begin{array}{l}\text { Inte } \\
\text { arc } \\
B\end{array}$ & $\begin{array}{l}\text { sity } \\
\text { park } \\
\text { S. }\end{array}$ & $\begin{array}{l}\text { Are intensity } \\
\text { and tempera- } \\
\text { ture class } \\
\mathbf{K} \text { and } \mathrm{C}\end{array}$ & $\begin{array}{l}\nu \text { (vac.) } \\
\mathrm{cm}^{-1}\end{array}$ & Zeeman effect & $\begin{array}{l}\text { Spec- } \\
\text { trum }\end{array}$ \\
\hline $\begin{array}{r}2,552.36 \\
46.40 \\
42.40 \\
41.60 \\
38.40\end{array}$ & $\frac{\overline{4}}{-1}$ & $\begin{array}{c}2 \\
20 \mathrm{hl} \\
6 \\
4 \\
2\end{array}$ & & $\begin{array}{r}39,167.68 \\
259.35 \\
321.11 \\
333.49 \\
383.07\end{array}$ & & $\begin{array}{l}\text { II } \\
\text { II } \\
\text { II } \\
\text { II } \\
\text { II }\end{array}$ \\
\hline $\begin{array}{l}36.76 \\
34.98 \\
33.14 \\
31.60 \\
30.26\end{array}$ & $\begin{array}{c}\overline{2} \\
10 \\
-\end{array}$ & $\begin{array}{r}3 \\
6 \\
15 \\
8 \\
1\end{array}$ & & $\begin{array}{l}408.53 \\
436.21 \\
464.84 \\
488.84 \\
509.76\end{array}$ & 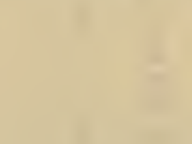 & $\begin{array}{l}\text { II } \\
\text { II } \\
\text { II } \\
\text { II } \\
\text { II }\end{array}$ \\
\hline $\begin{array}{l}27.84 \\
23.07 \\
19.22 \\
15.79 \\
14.59\end{array}$ & $\begin{array}{l}-1 \\
10 \\
-\end{array}$ & $\begin{array}{c}3 \\
5 \mathrm{hl} \\
50 \\
4 \\
3\end{array}$ & & $\begin{array}{l}547.58 \\
622.34 \\
682.89 \\
736.99 \\
755.95\end{array}$ & 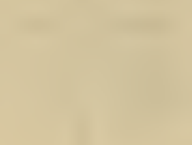 & $\begin{array}{l}\text { II } \\
\text { II } \\
\text { II } \\
\text { II } \\
\text { II }\end{array}$ \\
\hline $\begin{array}{r}2,501.18 \\
2,499.69 \\
95.82 \\
94.90 \\
87.59\end{array}$ & $\frac{1}{\frac{1}{10}}$ & $\begin{array}{c}15 \mathrm{hl} \\
1 \\
2 \\
1 \\
40\end{array}$ & & $\begin{array}{r}969.08 \\
39,992.91 \\
40,054.91 \\
069.68 \\
187.42\end{array}$ & & $\begin{array}{l}\text { II } \\
\text { II } \\
\text { II } \\
\text { II } \\
\text { II }\end{array}$ \\
\hline $\begin{array}{l}83.00 \\
79.85 \\
78.8 \\
76.72 \\
74.50 \\
72.44\end{array}$ & $\begin{array}{l}\frac{1}{5} \\
\overline{-} \\
\overline{1}\end{array}$ & $\begin{array}{c}5 \mathrm{hl} \\
10 \mathrm{l} \\
20 \mathrm{hl} \\
100 \mathrm{hl} \\
3 \\
10\end{array}$ & & $\begin{array}{l}261.71 \\
312.85 \\
329.9 \\
363.79 \\
400.00 \\
433.65\end{array}$ & & $\begin{array}{l}\text { II } \\
\text { II III } \\
\text { III }\end{array}$ \\
\hline $\begin{array}{l}71.90 \\
71.06 \\
70.55 \\
68.11 \\
58.15\end{array}$ & $\begin{array}{l}15 \\
= \\
=\end{array}$ & $\begin{array}{r}20 \\
5 \\
3 \\
1 \\
2\end{array}$ & & $\begin{array}{l}442.49 \\
456.23 \\
464.58 \\
501.58 \\
668.69\end{array}$ & 7 & $\begin{array}{l}\text { II } \\
\text { II } \\
\text { II } \\
\text { II } \\
\text { II }\end{array}$ \\
\hline $\begin{array}{l}55.88 \\
54.30 \\
52.73 \\
51.59 \\
45.56\end{array}$ & $\begin{array}{l}\bar{z} \\
\overline{1}\end{array}$ & $\begin{array}{c}10 \\
1 \\
8 \\
2 \\
10 \mathrm{~h}\end{array}$ & & $\begin{array}{l}706.28 \\
732.48 \\
758.55 \\
777.50 \\
878.04\end{array}$ & . & $\begin{array}{l}\text { II } \\
\text { II } \\
\text { II } \\
\text { II } \\
\text { II }\end{array}$ \\
\hline $\begin{array}{l}43.14 \\
42.80 \\
39.08 \\
38.42 \\
38.02\end{array}$ & $\begin{array}{l}\bar{z} \\
\overline{2}\end{array}$ & $\begin{array}{c}2 \mathrm{~h} \\
3 \\
2 \\
10 \\
20\end{array}$ & & $\begin{array}{r}918.53 \\
924.22 \\
986.63 \\
40,997.82 \\
41,004.45\end{array}$ & 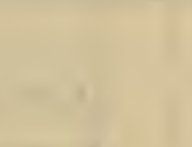 & $\begin{array}{l}\text { II } \\
\text { II } \\
\text { II } \\
\text { II } \\
\text { II }\end{array}$ \\
\hline $\begin{array}{l}37.14 \\
36.42 \\
31.40 \\
24.53 \\
21.61\end{array}$ & $\begin{array}{l}1 \\
1 \\
= \\
=\end{array}$ & $\begin{array}{c}10 \\
15 \\
6 \mathrm{~h} \\
2 \mathrm{~h} \\
5 \mathrm{~h}\end{array}$ & & $\begin{array}{l}019.26 \\
031.37 \\
116.08 \\
232.58 \\
282.29\end{array}$ & & $\begin{array}{l}\text { II } \\
\text { II } \\
\text { II } \\
\text { II } \\
\text { II }\end{array}$ \\
\hline $\begin{array}{l}20.01 \\
17.61 \\
12.08 \\
10.10 \\
07.79\end{array}$ & $\begin{array}{l}\frac{1}{z} \\
=\end{array}$ & $\begin{array}{l}5 \mathrm{hl} \\
3 \mathrm{~h} \\
2 \mathrm{~h} \\
5 \mathrm{hl} \\
5 \mathrm{hl}\end{array}$ & & $\begin{array}{l}309.58 \\
350.59 \\
445.38 \\
479.43 \\
519.22\end{array}$ & ' & $\begin{array}{l}\text { II } \\
\text { II } \\
\text { II } \\
\text { II } \\
\text { II }\end{array}$ \\
\hline $\begin{array}{r}04.65 \\
03.29 \\
2,401.46 \\
2,399.64 \\
98.70\end{array}$ & $\frac{-1}{3}$ & $\begin{array}{c}6 \\
7 \\
2 \mathrm{~h} \\
20 \mathrm{hl} \\
3 \mathrm{~h}\end{array}$ & & $\begin{array}{l}573.43 \\
596.96 \\
628.66 \\
660.22 \\
676.55\end{array}$ & & $\begin{array}{l}\text { II } \\
\text { II } \\
\text { II } \\
\text { II } \\
\text { II }\end{array}$ \\
\hline $\begin{array}{l}97.26 \\
94.98 \\
93.27 \\
89.84 \\
88.96\end{array}$ & $\begin{array}{l}\frac{1}{z} \\
=\end{array}$ & $\begin{array}{l}7 \mathrm{hl} \\
4 \\
2 \mathrm{~h} \\
3 \mathrm{hl} \\
2\end{array}$ & & $\begin{array}{l}701.57 \\
741.27 \\
771.10 \\
831.04 \\
846.45\end{array}$ & 41 & $\begin{array}{l}\text { II } \\
\text { II } \\
\text { II } \\
\text { II } \\
\text { II }\end{array}$ \\
\hline $\begin{array}{r}86.26 \\
84.28 \\
79.38 \\
75.63 \\
2,370.47\end{array}$ & $\frac{\overline{5}}{\overline{5 e}}$ & $\begin{array}{c}2 \\
3 \mathrm{~h} \\
200 \mathrm{~h} \\
2 \mathrm{~h} \\
2\end{array}$ & - & $\begin{array}{r}893.80 \\
41,928.58 \\
42,014.92 \\
081.24 \\
42,172.84\end{array}$ & & $\begin{array}{l}\text { II } \\
\text { II } \\
\text { II III } \\
\text { II }\end{array}$ \\
\hline
\end{tabular}


TABLE 1.-Wave lengths and Zeeman effects in lanthanum spectra-Continued

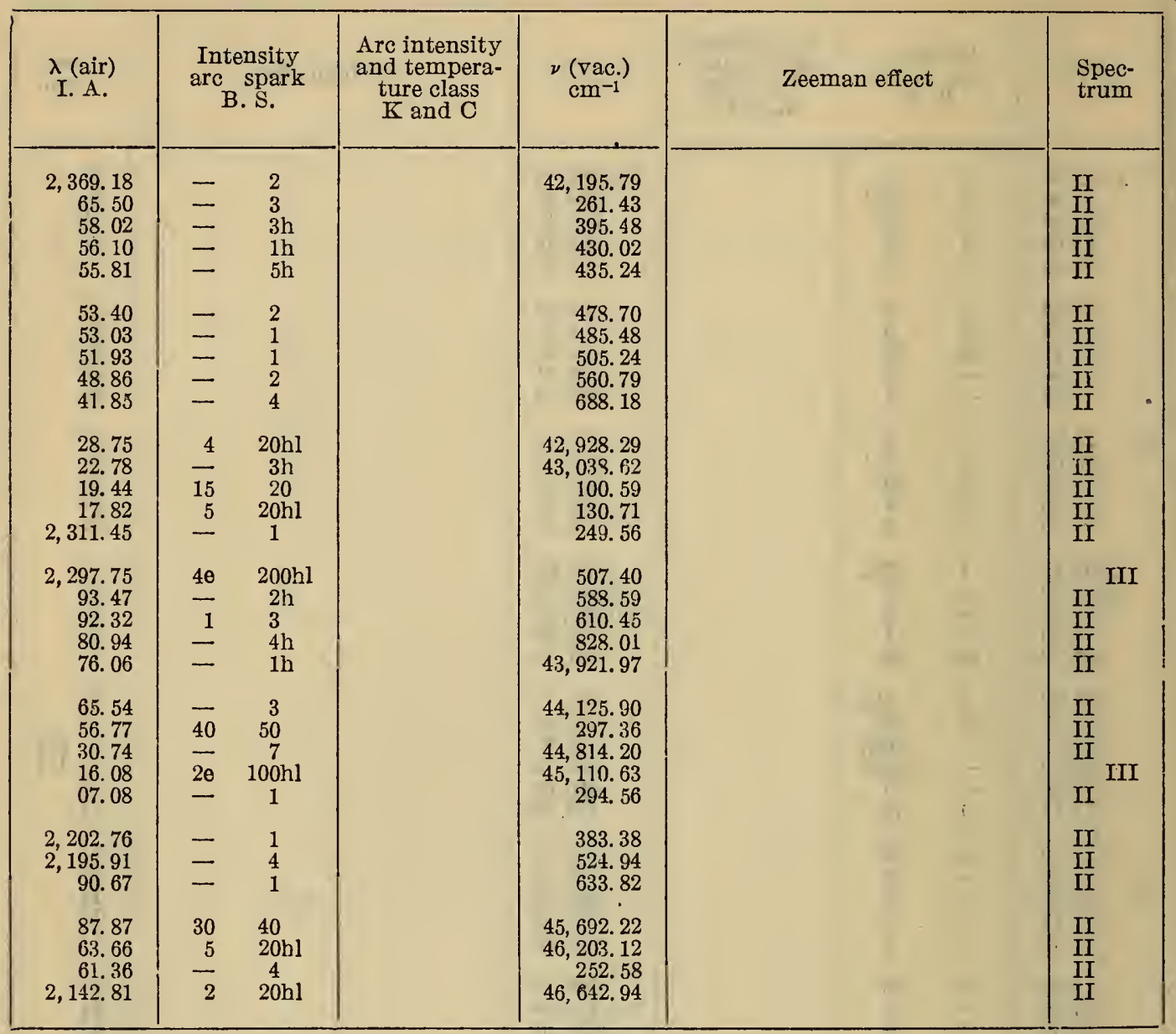

TABLE 2.-Band heads in the spectrum of lanthanum monoxide (LaO)

\begin{tabular}{|c|c|c|c|}
\hline$\lambda$ air I. A. & $\begin{array}{l}\text { Inten- } \\
\text { sity }\end{array}$ & $\nu \operatorname{vac} \mathrm{cm}-1$ & $\underset{v^{\prime}, v^{\prime \prime}}{\text { System }}$ \\
\hline $\begin{array}{l}8,423.3 \\
8,994.5 \\
9,035.9 \\
9,073.4 \\
9,111.5\end{array}$ & $\begin{array}{l}2 \\
3 \\
1 \\
1 \\
2\end{array}$ & $\begin{array}{l}11,868.6 \\
11,11.9 \\
11,063.9 \\
11,018.2 \\
10,972.1\end{array}$ & $\begin{array}{lr}\text { VII } & 14,14 \\
\text { VII } & 14,15 \\
\text { VII } & 15,16 \\
\text { VII } & 0,2 \\
\text { VII } & 1,3\end{array}$ \\
\hline $\begin{array}{l}9,150.1 \\
9,188.8 \\
9,228.15 \\
9,267.8 \\
9,307.8\end{array}$ & $\begin{array}{l}2 \\
4 \\
4 \\
4 \\
4\end{array}$ & $\begin{array}{l}925.9 \\
879.8 \\
833.4 \\
787.1 \\
740.7\end{array}$ & $\begin{array}{lll}\text { VII } & 2,4 \\
\text { VII } & 3,5 \\
\text { VII } & 4,6 \\
\text { VII } & 5,7 \\
\text { VII } & 6,8\end{array}$ \\
\hline $\begin{array}{l}9,348.2 \\
9,388.8 \\
9,429.9 \\
9,471.4 \\
9,513.3\end{array}$ & $\begin{array}{l}3 \\
3 \\
3 \\
3 \\
3 \\
3\end{array}$ & $\begin{array}{l}694.3 \\
648.1 \\
601.7 \\
555.2 \\
508.7\end{array}$ & $\begin{array}{lr}\text { VII } & 7,9 \\
\text { VII } & 8,10 \\
\text { VII } & 9,11 \\
\text { VII } & 10,12 \\
\text { VII } & 11,13\end{array}$ \\
\hline $\begin{array}{l}9,555.5 \\
9,582.2 \\
9,641.1 \\
9,684.8 \\
9,729.1\end{array}$ & $\begin{array}{l}3 \\
3 \\
3 \\
3 \\
3\end{array}$ & $\begin{array}{r}462.3 \\
415.8 \\
369.4 \\
322.6 \\
10,275.6\end{array}$ & $\begin{array}{ll}\text { VII } & 12,14 \\
\text { VII } & 13,15 \\
\text { VII } & 14,16 \\
\text { VII } & 15,17 \\
\text { VIII } & 16,18\end{array}$ \\
\hline
\end{tabular}

Washington, June 11, 1932. 